\title{
$K$-THEORY OF SEMI-LOCAL RINGS WITH FINITE COEFFICIENTS AND ÉTALE COHOMOLOGY
}

\author{
BRUNO KAHN
}

\section{Contents}

1. Statement of results 1

2. Construction of $B_{A}^{n} \quad 6$

3. Proofs of theorem 1, theorem 2 and corollary $1 \quad 10$

4. Higher Chern classes with values in truncated étale cohomology 18

5. Proof of theorem $3 \quad 24$

$\begin{array}{ll}\text { References } & 27\end{array}$

Abstract. Let $A$ be a commutative semi-local ring containing $1 / 2$. We construct natural isomorphisms

$$
\coprod_{0 \leq i \leq n} H_{\text {ét }}^{2 i-n}\left(A, \mu_{2^{\nu}}^{\otimes i}\right) \stackrel{\sim}{\longrightarrow} K_{n}\left(A, \mathbf{Z} / 2^{\nu}\right) \quad(\nu \geq 1)
$$

if either $A$ is local and $\sqrt{-1} \in A$, or char $A>2$. We deduce that, for a scheme $X$ quasiprojective or regular over $\mathbf{Z}[1 / 2, i]$ or $\mathbf{F}_{p}(p \neq 2)$, the groups $K_{n}\left(X, \mathbf{Z} / 2^{\nu}\right)$ and $K_{n}^{\prime}\left(X, \mathbf{Z} / 2^{\nu}\right)$ are finite for $n \geq \operatorname{dim}(X)-1$. Finally, using higher Chern classes with values in truncated étale cohomology, we show that, for $X$ over $\mathbf{Z}[1 / 2]$, of Krull dimension $d$, quasiprojective over an affine base (resp. smooth over a field or a discrete valuation ring), $K_{n}\left(X, \mathbf{Z} / 2^{\nu}\right)$ is isomorphic for $n \geq$ 3 (resp. for $n \geq 2$ ) to $\coprod_{i \geq 1} H_{\mathrm{Zar}}^{2 i-n}\left(X, \tau_{\leq i} R \alpha_{*} \mu_{2^{\nu}}^{\otimes i}\right)$, up to controlled torsion depending only on $n$ and $d$ (not on $\nu$ ). Here $\alpha$ is the projection from the étale site of $X$ to its Zariski site and $\tau$ denotes truncation in the derived category.

\section{Statement of Results}

The aim of this article is to prove the following theorems and corollary:

Theorem 1. Let $A$ be a commutative semi-local ring such that 2 is invertible in A. Suppose that

- $A$ is local and $\sqrt{-1} \in A$

or

- $A$ is an $\mathbf{F}_{p}$-algebra for some $p>2$.

Date: April 27, 1999. 
Then,

a) For all $n \geq 0$ and $\nu \geq 1$, there exists an isomorphism

$$
\coprod_{0 \leq i \leq n} H_{\text {ét }}^{2 i-n}\left(A, \mu_{2^{\nu}}^{\otimes i}\right) \stackrel{B_{A}^{n}}{\sim} K_{n}\left(A, \mathbf{Z} / 2^{\nu}\right)
$$

which is natural in $A$.

b) For all $\nu \geq 1$, the ring $K_{*}\left(A, \mathbf{Z} / 2^{\nu}\right)$ is multiplicatively generated by units and the Bott element, up to transfer.

c) If $A$ is a field $F$, then for all $n, \nu \geq 0$, the natural map $K_{n}^{M}(F) / 2^{\nu} \rightarrow K_{n}(F) / 2^{\nu}$ is split injective.

d) If $A$ is a field $F$ and $\mu_{2^{\nu}} \subset F$, then the natural ring homomorphism

$$
K_{*}^{M}(F) / 2^{\nu}[t] \rightarrow K_{*}\left(F, \mathbf{Z} / 2^{\nu}\right)
$$

mapping t to a Bott element in $K_{2}\left(F, \mathbf{Z} / 2^{\nu}\right)$ is an isomorphism.

Note that the assumptions on $A$ imply that the product on $K_{*}\left(A, \mathbf{Z} / 2^{\nu}\right)$ is defined, associative and commutative for all $\nu \geq 1, c f$. [6].

Part a) of this theorem is a confirmation of the main conjecture in [26] for $l=2$ and the cases given. It even removes the regularity assumption of loc. cit.. It was earlier obtained in [28, th. 4.1] for $A$ a higher local field in the sense of Kato (and $2^{\nu}$ replaced by $l^{\nu}, l$ any prime number different from the "essential" residue characteristic of $A$ ). Part b) is a variant of a conjecture of Suslin [45, conj. 4.1], and part c) extends [26, th. 3] to all values of $n$.

The next theorem settles the Dwyer-Friedlander-Snaith-Thomason version of the Quillen-Lichtenbaum conjecture [12, top p. 482] in the positive for 2-primary coefficients, improving on earlier results of Thomason ([49]; see [37, §6] for the original statement of this conjecture). For any scheme $X$, define

$$
d_{2}(X)=\sup \left\{c d_{2}(\eta)\right\}
$$

where $\eta$ runs through the generic points of $X$ and $c d_{2}$ denotes the étale cohomological dimension.

Theorem 2. Let $X$ be a finite-dimensional Noetherian $\mathbf{Z}[1 / 2]$-scheme whose residue fields either contain $\sqrt{-1}$ or are of characteristic $>2$. Then

a) The natural map

$$
K_{n}^{T T}\left(X, \mathbf{Z} / 2^{\nu}\right) \rightarrow K_{n}^{T T}\left(X, \mathbf{Z} / 2^{\nu}\right)\left[\beta^{-1}\right]
$$

is injective for $n \geq \sup \left(d_{2}(X)-2,1\right)$ and bijective for $n \geq \sup \left(d_{2}(X)-1,1\right)$. The 1 in the sup is not necessary if $X$ is regular.

b) The natural map

$$
K_{n}^{\prime}\left(X, \mathbf{Z} / 2^{\nu}\right) \rightarrow K_{n}^{\prime}\left(X, \mathbf{Z} / 2^{\nu}\right)\left[\beta^{-1}\right]
$$


is injective for $n \geq d_{2}(X)-2$ and bijective for $n \geq d_{2}(X)-1$.

c) If $c d_{2}(X)<+\infty$, there are isomorphisms

$$
K_{n}^{T T}\left(X, \mathbf{Z} / 2^{\nu}\right)\left[\beta^{-1}\right] \stackrel{\sim}{\rightarrow} K_{n}^{\text {ét }}\left(X, \mathbf{Z} / 2^{\nu}\right)
$$

for all $n \in \mathbf{Z}$.

Here $K_{n}^{T T}\left(X, \mathbf{Z} / 2^{\nu}\right)$ denotes the Thomason-Trobaugh algebraic $K$-theory of perfect complexes on $X$ with finite coefficients $[51, \S 6], K_{*}^{T T}\left(X, \mathbf{Z} / 2^{\nu}\right)\left[\beta^{-1}\right]$ denotes the corresponding $K$-theory with the Bott element inverted (ibid., 11.4), $K_{n}^{\prime}\left(X, \mathbf{Z} / 2^{\nu}\right)$ denotes Quillen's $K^{\prime}$-theory of coherent sheaves and $K_{n}^{\text {ét }}\left(X, \mathbf{Z} / 2^{\nu}\right)$ denotes the nonconnective version of the étale $K$-theory of Dwyer-Friedlander [11].

Recall that Thomason-Trobaugh's $K$-theory coincides with Quillen's algebraic $K$-theory when $X$ has an ample family of line bundles (e.g. $X$ quasi-projective over an affine base or regular) by [51, th. 7.6]. c) generalises a theorem of Thomason [48, th. 4.11] which holds under much more restrictive hypotheses (in particular, that $X$ is regular and that its residue fields have a "Tate-Tsen filtration").

The corollary which follows is closely related to results of Colliot-Thélène-SansucSoulé on the finiteness of torsion in $\mathrm{CH}^{2}$ of certain varieties [9].

Corollary 1. Let $S$ be a scheme and $X$ a separated scheme of finite type over $S$. Assume that $S$ is of one of the following types:

(i) $\operatorname{Spec} \mathbf{Z}[1 / 2, \sqrt{-1}]$.

(ii) $\operatorname{Spec} \mathbf{F}_{p}, p>2$.

(iii) Spec $k, k$ a separably closed field of characteristic $\neq 2$.

(iv) Spec $k, k$ a higher local field in the sense of Kato.

Then $K_{n}^{T T}\left(X, \mathbf{Z} / 2^{\nu}\right)$ is finite for $n \geq \operatorname{dim}(X / S)+c d_{2}(S)-2$, except perhaps in the case (iii), $n=0$ and $X$ a singular surface. The group $K_{n}^{\prime}\left(X, \mathbf{Z} / 2^{\nu}\right)$ is finite for $n \geq \operatorname{dim}(X / S)+c d_{2}(S)-2$.

Remark. Actually, $K_{0}^{T T}\left(X, \mathbf{Z} / 2^{\nu}\right)$ is also finite in the exceptional case, i.e. $X$ a singular surface over a separably closed field [56].

In the last result, we consider higher Chern classes $c_{i, j}$ with values in truncated étale cohomology (compare [2, 5.10 C (vi)]); these Chern classes are defined in section 4 .

Theorem 3. Let $d \geq 0$ and $n \geq 3$. Then there exists an effectively computable integer $N=N(d, n)>0$ such that, for any Noetherian $\mathbf{Z}[1 / 2]$-scheme $X$ separated of Krull dimension $\leq d$ and for all $\nu \geq 2$, the kernel and cokernel of the map

$$
K_{n}^{T T}\left(X, \mathbf{Z} / 2^{\nu}\right) \stackrel{\left(i c_{i, 2 i-n}\right)}{\longrightarrow} \prod_{i \geq 1} H_{\mathrm{Zar}}^{2 i-n}\left(X, B / 2^{\nu}(i)\right)
$$

are killed by $N$. If $X$ is smooth over a field or a discrete valuation ring, this holds also for $n=2$. 
Here $B / 2^{\nu}(i)=\tau_{\leq i} R \alpha_{*} \mu_{2^{\nu}}^{\otimes i}$, where $\alpha$ is the projection of the big étale site of $\operatorname{Spec} \mathbf{Z}[1 / 2]$ onto its big Zariski site, as in [47]. Using Newton polynomials in the Chern classes, one can expect to extend this result to $n=0$, although we haven't done it; we don't know what happens for $n=1$.

Theorem 3 is related to a conjecture of Beilinson [2, 5.10 B and C (vi)], asserting that there should exist a spectral sequence of cohomological type, at least for regular $X$

$$
E_{2}^{p, q}=H_{\mathrm{Zar}}^{p-q}\left(X, B / 2^{\nu}(-q)\right) \Rightarrow K_{-p-q}\left(X, \mathbf{Z} / 2^{\nu}\right)
$$

which would be split, up to small torsion (another variant of the Quillen-Lichtenbaum conjecture). This spectral sequence is now constructed at least for $X$ regular over a regular base of dimension $\leq 1$ [32] (see section 3 for more details), and one can expect that the Chern classes of theorem 3 actually split it with the usual small factorials.

To prove theorems 1, 2 and 3, we use (1.3) only for fields. As a by-product, we get that (1.3) degenerates completely and canonically for fields under the conditions of theorem 1. What we need as a crucial tool is the existence of a product structure on this spectral sequence. A construction of this product structure was performed only recently by Marc Levine [33] and also by Friedlander-Suslin (in preparation), thanks to the latter's reinterpretation of the Bloch-Lichtenbaum spectral sequence. To the best of our efforts, we haven't been able to find an argument avoiding it. This explains the long delay between the first version of this paper and its current revision.

Some further comments are appropriate:

1. The condition $\sqrt{-1} \in A$ in theorem 1 cannot be removed, as the example $A=\mathbf{R}$ shows [44]. On the other hand, it can probably be weakened to $A$ being "non-exceptional" in the sense of [26], which would cover both cases of the theorem. This would require to extend [29, cor. 9.5] to this case.

2. It is not clear how to extend theorem 1 a) to Bass' negative $K$-groups with 2-primary coefficients. As stated, the result is certainly wrong for $n<0$ as the left hand side is 0 and there are examples where the right hand side is not $[54 \text {, ex. 8.5 }]^{1}$.

3. One could naïvely hope that the isomorphisms (1.1) globalize into similar isomorphisms involving truncated étale cohomology groups, letting (1.3) degenerate on the nose, as in the proof of theorem 1 a) for fields. However this is unreasonable. A special case of such isomorphisms would be a canonical decomposition $K_{0}(X) / 2^{\nu} \simeq \coprod_{i \geq 0} C H^{i}(X) / 2^{\nu}$ for any smooth variety $X$ over

\footnotetext{
${ }^{1}$ More precisely, for all $d \geq 1 \mathrm{~L}$. Reid gives in [38] an example of a $d$-dimensional affine normal scheme $X$ with exactly one singular point $P$, such that $K_{-d}(X)$ admits a nontrivial homomorphism to Z . For $d>1$, C. Weibel proves in $[53,1.6]$ that $K_{-d}(X) \stackrel{\sim}{\longrightarrow} K_{-d}\left(X^{h}\right)$, where $X^{h}$ is the Henselisation of $X$ at $P$. Therefore $K_{-d}\left(X^{h}\right)$ admits a nontrivial homomorphism to $\mathbf{Z}$, hence $K_{-d}\left(X^{h}\right) / 2^{\nu} \neq 0$ and $K_{-d}\left(X^{h}, \mathbf{Z} / 2^{\nu}\right) \neq 0$.
} 
a field, a fact for which counterexamples exist. Over $\mathbf{C}$, comparison with the Atiyah-Hirzebruch spectral sequence for topological $K$-theory also yields examples where the Thomason descent spectral sequence of [48] does not degenerate, which strongly suggests that (1.3) should not degenerate in general (compare $[50, \S 8]$ ). Theorem 1 a) does contain nontrivial information on (1.3), but exactly what information is not clear at this stage.

4. It may seem singular that our results hold even for non-smooth schemes. This is thanks to both Hoobler's Henselian pair trick [24] and the ThomasonTrobaugh descent theorem for the Bass extension of algebraic $K$-theory [51]. Theorem 3 suggests that a form of (1.3) exists even for singular schemes (I write a form because of negative $K$-groups, which remain mysterious at this stage), and also that Voevodsky's cdh cohomology (e.g. [47]) will not be necessary in the end.

5. The Bass conjecture implies the finiteness of $K_{n}\left(X, \mathbf{Z} / 2^{\nu}\right)$ for all $n$ when $X$ is regular of finite type over $\mathbf{Z}$; corollary 1 suggests this should hold more generally without a regularity assumption, provided $X$ is over $\mathbf{Z}[1 / 2]$. For $X$ regular, this would be a consequence of (1.3) plus the finiteness of the groups $H_{\text {Zar }}^{p-q}\left(X, B / 2^{\nu}(-q)\right)$, which at this point is known only for $p \leq 1$ by reduction to étale cohomology. The latter finiteness for all $p$, in turn, is essentially equivalent to the finiteness of the groups $H_{\mathrm{Zar}}^{p}\left(X, R^{q} \alpha_{*} \mu_{2^{\nu}}^{\otimes i}\right)$. It is probably wrong for $X$ of finite type over an algebraically closed field in general: $C$. Schoen has produced an example of a 3 -dimensional variety $X$ over $\overline{\mathbf{Q}}$ such that $C H^{2}(X) / l$ is infinite for a suitable prime $l$.

6 . The reader should beware that most of the above is wrong if we work with integral algebraic $K$-theory:

- Theorem 3 implies that the Beilinson-Soulé vanishing conjecture holds for the $K$-theory of singular schemes with 2-primary coefficients, in a sense that could be made more precise using Adams operations. However, Feigin-Tsygan [15] and independently Geller-Weibel [19] have produced examples of singular varieties whose integral $K$-theory fails to verify the Beilinson-Soulé conjecture.

- The integral analogue of corollary 1 is expected to hold for regular schemes of finite type over $\mathbf{Z}$ (the Bass conjecture) but is known to be false for singular ones, as the famous example $\mathbf{Z}[T, U] / U^{2}$ shows for $K_{1}$.

- As shown by Rob de Jeu [25], the integral analogue of theorem $1 \mathrm{~b}$ ) is false for fields of characteristic 0 in general, already for $K_{4}$. (However we show in [30, appendix A] that, for fields of characteristic $>0$, it follows from the Bass conjecture.)

7. The proofs of theorems 1,2 and 3 rest, inter alia, on Voevodsky's affirmation of the Milnor conjecture [52] and the related Suslin-Voevodsky and GeisserLevine results [47], [20]. 
8. Since the spectral sequence (1.3) is multiplicative for schemes as in theorem 2 and regular, one can invert the Bott element in it, getting a spectral sequence closely related (probably isomorphic) to the Thomason spectral sequence. Moreover, the Beilinson-Lichtenbaum conjecture with 2-primary coefficients (which follows from the Milnor conjecture, see [47] and [20]) implies that the map between (1.3) and this latter spectral sequence is an isomorphism on the $E_{2}$-terms in a range. This yields a much more direct proof of theorem 2 and corollary 1 for $X$ regular. But in the case of non-regular schemes, I don't see that one can construct (1.3) without using a strong form of resolution of singularities in nonzero or mixed characteristics.

9. Needless to say, the arguments in this paper will apply by replacing 2 by an odd prime $l$ once the Bloch-Kato conjecture is proven for $l$, yielding the same results (without the restrictions on $\sqrt{-1}$ ).

\section{Construction of $B_{A}^{n}$}

Proposition 2.1. For any commutative $\mathbf{Z}[1 / 2]$-algebra $A$, let

$$
H_{n}(A)=\coprod_{0 \leq i \leq n} H_{\text {ét }}^{2 i-n}\left(\operatorname{Spec} A, \mu_{2^{\nu}}^{\otimes i}\right) .
$$

Then there exists a unique collection of natural transformations

$$
B_{A}^{n}: H_{n}(A) \rightarrow K_{n}\left(A, \mathbf{Z} / 2^{\nu}\right)
$$

defined on the category of commutative semi-local rings as in theorem 1 and having the following properties:

(i) They are compatible when $\nu$ varies.

(ii) They are multiplicative.

(iii) They commute with transfer.

(iv) If $A$ is a dvr with quotient field $E$ and residue field $F$, then the diagram

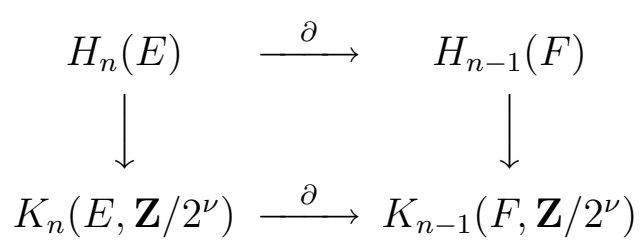

commutes, where the horizontal maps are residue homomorphisms in étale cohomology and algebraic $K$-theory.

(v) (normalization) If $A$ is a field $F$, then $B_{F}^{0}$ is the map $1 \mapsto[F], B_{F}^{1}$ is induced by the inclusion $G L_{1}(F) \hookrightarrow G L(F)$ via Kummer theory and $B_{F}^{2}$ restricted to $H^{0}\left(F, \mu_{2^{n}}\right)$ is given by the Bott element construction.

There is a similar collection of natural transformations

$$
B_{A}^{n \text {,ét }}: H_{n}^{\prime}(A) \rightarrow K_{n}\left(A, \mathbf{Z} / 2^{\nu}\right)\left[\beta^{-1}\right]
$$


where $H_{n}^{\prime}(A)=\coprod_{0 \leq i} H_{\text {ét }}^{2 i-n}\left(\operatorname{Spec} A, \mu_{2^{\nu}}^{\otimes i}\right)$, enjoying the same properties; the diagrams

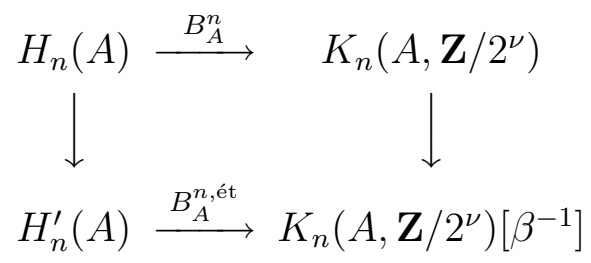

commute.

Proof. It goes in four steps:

I) $A$ is a field $F$, where $\sqrt{-1} \in F$ or $\operatorname{char} F>2$;

II) $A$ is a (semi-)localization of a smooth $R$-scheme, where $R$ is a field or a discrete valuation ring;

III) $A$ is a (semi-)localization of a not necessarily smooth $R$-scheme, where $R$ is as in II);

IV) the general case.

I) Fields. We first recall from [26] and [28] the construction of "anti-Chern classes"

$$
\begin{aligned}
& H_{\text {ét }}^{j}\left(F, \mu_{2^{\nu}}^{\otimes i}\right) \stackrel{\beta_{F}^{i, j}}{\longrightarrow} K_{2 i-j}\left(F, \mathbf{Z} / 2^{\nu}\right) \quad(i \geq j) \\
& H_{\text {ét }}^{j}\left(F, \mu_{2^{\nu}}^{\otimes i}\right) \stackrel{\tilde{\beta}_{F}^{i, j}}{\longrightarrow} K_{2 i-j}\left(F, \mathbf{Z} / 2^{\nu}\right)\left[\beta^{-1}\right](i \in \mathbf{Z})
\end{aligned}
$$

from étale cohomology to algebraic $K$-theory and Bott-localised algebraic $K$-theory; this construction uses the Milnor conjecture, the main results of [27] and [29] and was already given in [26] and [28] (in the former, assuming [27] and [29]), to which we refer for more details. Recall the twisted Milnor $K$-groups of $[28, \S 1]$

$$
K_{j}^{M}(i)\left(F, \mathbf{Z} / 2^{\nu}\right)=\left(K_{j}^{M}\left(F\left(\mu_{2^{\nu}}^{\otimes i}\right)\right) \otimes \mu_{2^{\nu}}^{\otimes i}\right)_{G_{i}} \quad(j \geq 0, i \in \mathbf{Z})
$$

where $G_{i}=\operatorname{Gal}\left(F\left(\mu_{2^{\nu}}^{\otimes i}\right) / F\right)$. In particular, $K_{j}^{M}(0)\left(F, \mathbf{Z} / 2^{\nu}\right)=K_{j}^{M}(F) / 2^{\nu}$. Set $E=F\left(\mu_{2^{\nu}}^{\otimes i-j}\right)$ and $G=G_{i-j}$. We define composite homomorphisms

$$
\begin{aligned}
u_{F}^{i-j, j}: K_{j}^{M}(i-j)\left(F, \mathbf{Z} / 2^{\nu}\right) \stackrel{u_{E}^{0, j} \otimes 1}{\longrightarrow}\left(H_{\text {ét }}^{j}(E\right. & \left.\left., \mu_{2^{\nu}}^{\otimes j}\right) \otimes \mu_{2^{\nu}}^{\otimes(i-j)}\right)_{G} \\
& =H_{\text {ét }}^{j}\left(E, \mu_{2^{\nu}}^{\otimes i}\right)_{G} \stackrel{\text { Cor }}{\longrightarrow} H_{\text {ét }}^{j}\left(F, \mu_{2^{\nu}}^{\otimes i}\right)
\end{aligned}
$$

where $u_{E}^{0, j}$ is the Galois symbol of degree $j$. (Note that the restriction $i \geq 2 j$ from $[28,(2.2)]$ is irrelevant.) We have the following proposition, which follows from the Milnor conjecture in degree $j$ and the main result of [27]:

Proposition 2.2 (cf. [27, th. 1(2)]). Under the assumptions of theorem 1, the map

$$
H_{\text {ét }}^{j}\left(E, \mu_{2^{\nu}}^{\otimes i}\right)_{G} \stackrel{\text { Cor }}{\longrightarrow} H_{\text {ét }}^{j}\left(F, \mu_{2^{\nu}}^{\otimes i}\right)
$$

is an isomorphism. 
This proposition (together with the Milnor conjecture) implies that $u_{F}^{i-j, j}$ is an isomorphism for all $i$.

Next, we have homomorphisms

$$
\begin{array}{ll}
K_{j}^{M}(i-j)\left(F, \mathbf{Z} / 2^{\nu}\right) & \stackrel{\eta_{F}^{i, 2 i-j}}{\longrightarrow} \\
K_{j}^{M}(i-j)\left(F, \mathbf{Z} / 2^{\nu}\right) \stackrel{\tilde{\eta}_{F}^{i, 2 i-j}}{\longrightarrow} & K_{2 i-j}\left(F, \mathbf{Z} / 2^{\nu}\right) \\
K_{2 i-j}\left(F, \mathbf{Z} / 2^{\nu}\right)\left[\beta^{-1}\right]
\end{array}
$$

where $\eta_{F}^{i, 2 i-j}$ is defined for $i \geq j$, while $\tilde{\eta}_{F}^{i, 2 i-j}$ is defined for all $i \in \mathbf{Z}$, constructed as compositions

$$
\begin{aligned}
K_{j}^{M}(i-j)\left(F, \mathbf{Z} / 2^{\nu}\right) \stackrel{\eta_{E}^{j, j} \otimes \eta_{E}^{i-j, 2(i-j)}}{\longrightarrow}\left(K_{j}\left(E, \mathbf{Z} / 2^{\nu}\right) \otimes K_{2(i-j)}\left(E, \mathbf{Z} / 2^{\nu}\right)\right)_{G} \\
\stackrel{\mu}{\longrightarrow} K_{2 i-j}\left(E, \mathbf{Z} / 2^{\nu}\right)_{G} \stackrel{N}{\longrightarrow} K_{2 i-j}\left(F, \mathbf{Z} / 2^{\nu}\right)
\end{aligned}
$$

and similarly for étale $K$-theory. Here $\eta_{E}^{j, j}$ is the natural mapping of Milnor's $K$ theory to Quillen's $K$-theory, $\eta_{E}^{i-j, 2(i-j)}$ is the canonical Bott element mapping of [29], $\mu$ is product and $N$ is the norm map (compare [28, prop. 1.2 and its proof]). For $i \geq j, \eta_{F}^{i, 2 i-j}$ and $\tilde{\eta}_{F}^{i, 2 i-j}$ commute with the canonical map $K_{2 i-j}\left(F, \mathbf{Z} / 2^{\nu}\right) \rightarrow$ $K_{2 i-j}\left(F, \mathbf{Z} / 2^{\nu}\right)\left[\beta^{-1}\right]$.

The anti-Chern classes $\beta_{F}^{i, j}$ and $\tilde{\beta}_{F}^{i, j}$ are then defined as $\eta_{F}^{i, 2 i-j} \circ\left(u_{F}^{i-j, j}\right)^{-1}$ and $\tilde{\eta}_{F}^{i, 2 i-j} \circ\left(u_{F}^{i-j, j}\right)^{-1}$. The maps $B_{F}^{n}$ and $B_{F}^{n \text {,ét }}$ are defined from the $\beta_{F}^{i, j}$ and $\tilde{\beta}_{F}^{i, j}$ componentwise. They are natural in $F$, since the $\beta_{F}^{i, j}$ and $\tilde{\beta}_{F}^{i, j}$ are.

In the sequel, we only construct the maps $B_{A}^{n}$ for more general $A$; the case of $B_{A}^{n \text {,ét }}$ is completely similar.

II) Smooth (semi-)local rings. Let $E$ be the field of fractions of $A$. There is a commutative diagram of exact sequences:

$$
\begin{aligned}
& 0 \rightarrow H_{n}(A) \quad \longrightarrow \quad H_{n}(E) \quad \longrightarrow \quad \coprod_{x \in X^{(1)}} H_{n-1}(\kappa(x)) \\
& B_{E}^{n} \downarrow \quad\left(B_{\kappa(x)}^{n-1}\right) \downarrow \\
& 0 \longrightarrow K_{n}\left(A, \mathbf{Z} / 2^{\nu}\right) \longrightarrow K_{n}\left(E, \mathbf{Z} / 2^{\nu}\right) \longrightarrow \coprod_{x \in X^{(1)}} K_{n-1}\left(\kappa(x), \mathbf{Z} / 2^{\nu}\right)
\end{aligned}
$$

where $X=\operatorname{Spec} A, \kappa(x)$ is the residue field at $x \in X$ and and $X^{(1)}$ denotes the set of points of codimension 1 . In case $R$ is a field, the bottom exact sequence follows from [36, th. 7.5.11] and the top one from [5]. In case $R$ is a dvr, the bottom exact sequence is Gersten's conjecture for $K$-theory with finite coefficients for essentially smooth local rings over a discrete valuation ring, due to Gillet and Levine [23]; the top exact sequence is due to Gillet [22]. To see that the diagram commutes, one reduces to showing that similar diagrams involving the maps $\eta^{i, j}$ and $u^{i, j}$ commute; this in turn reduces to easy special cases since the source groups are then defined 
by generators and relations. Note that, if $\sqrt{-1} \in A$, then $\sqrt{-1} \in E, \kappa(x)$ for all $x$. The diagram defines in a unique way a homomorphism $B_{A}^{n}: H_{n}(A) \rightarrow K_{n}\left(A, \mathbf{Z} / 2^{\nu}\right)$. We note that, by construction, $B_{A}^{n}$ is natural in $A$ when $A$ varies in the category of essentially smooth (semi-)local $R$-algebras.

III) (Semi-)local rings of "geometric" origin. We use a method of Hoobler [24]: there exists a henselian pair $(B, I)$ such that

- $B / I=A$

- $B$ is a union of (semi-)localizations of smooth $R$-schemes.

(To see this, write $A$ as the (semi-)localization of some finitely generated $R$ algebra $A_{0}$ at a prime ideal $\mathfrak{p}$ (resp. at a finite set of prime ideals $S$ ), and write $A_{0}$ as a quotient of $B_{0}=R\left[T_{1}, \ldots, T_{n}\right]$ for $n$ large enough. Then $A$ is a quotient of the local ring $B_{1}:=\left(B_{0}\right)_{\mathfrak{p} \cap B_{0}}$ (resp. $\left.B_{1}:=\left(B_{0}\right)_{S \cap B_{0}}\right)$. We take for $(B, I)$ the henselization of the pair $\left(B_{1}, \operatorname{Ker}\left(B_{1} \rightarrow A\right)\right)$.)

Since both algebraic $K$-theory and étale cohomology commute with filtering inverse limits of schemes with affine transition morphisms, and since the homomorphism $B^{n}$ constructed in II) is natural, we deduce from II) a homomorphism $B_{B}^{n}: H_{n}(B) \rightarrow K_{n}\left(B, \mathbf{Z} / 2^{\nu}\right)$. There is a diagram

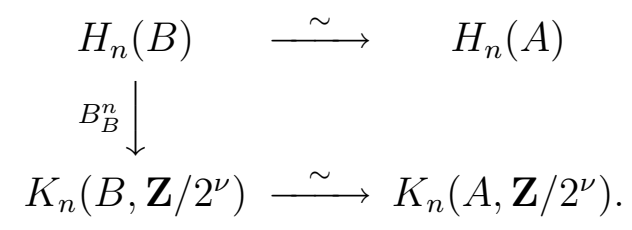

The top horizontal map is an isomorphism by a theorem of Gabber [16] and independently Strano [43]. The bottom one is also an isomorphism, by another theorem of Gabber [17]. Note that, if $\sqrt{-1} \in A$, then $\sqrt{-1} \in B$, since $(B, I)$ is a henselian pair. Hence the diagram again defines a homomorphism $B_{A}^{n}: H_{n}(A) \rightarrow$ $K_{n}\left(A, \mathbf{Z} / 2^{\nu}\right)$, which is natural in the category of local $R$-algebras essentially of finite type.

IV) The general case. Write $A$ as a direct limit of its finitely generated subalgebras. After localizing the latter, we write $A$ as a direct $\operatorname{limit} \underline{\lim } A_{i}$, where the $A_{i}$ are as in III), with $R$ a suitable (semi-)localization of $\mathbf{Z}[1 / 2]$. Note that, if $\sqrt{-1} \in A$, then $\sqrt{-1} \in A_{i}$ for $i$ large enough. By naturality, we then get the desired homomorphism $B_{A}^{n}$ as the direct limit of the $B_{A_{i}}^{n} \mathrm{~s}$. And $B_{A}^{n}$ is clearly natural in $A$.

Properties (i)-(iv) of proposition 2.1 now follow from the definition of the antiChern classes and [29, cor. 9.5]. As for uniqueness, the method of construction shows that $B^{n}$ is determined by its value on fields. By the Milnor conjecture and $[27$, th. $1(2)]$, the bigraded ring $H^{*}\left(F, \mu_{2^{\nu}}^{\otimes *}\right)$ is generated by $H^{0}\left(F, \mu_{2^{\nu}}\right)$ and 
$H^{1}\left(F, \mu_{2^{\nu}}\right)$ modulo transfer. Hence $B^{n}$ is determined by the value of $B^{0}, B^{1}$ and $B^{2}$ on fields.

The commutation of the diagrams at the end of proposition 2.1 is clear for fields by construction; the general case is obtained by going through II), III) and IV) as before.

Protoglobalization. We have the following variant of proposition 2.1:

Proposition 2.3. For all $n, \nu \geq 0$, there is a unique commutative diagram of homomorphism of sheaves over the big Zariski sites of $\operatorname{Spec} \mathbf{Z}[1 / 2, i]$ and $\operatorname{Spec} \mathbf{F}_{p}$, $p>2$ :

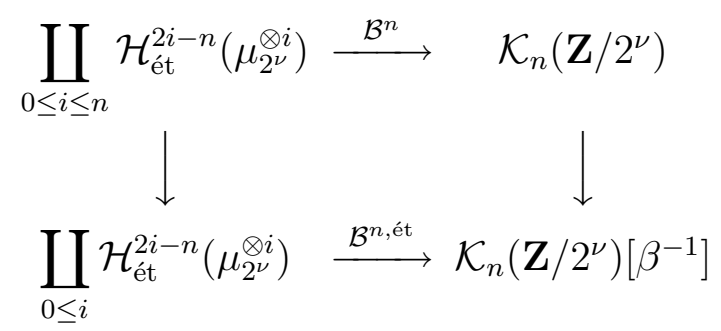

which coincides with $B^{n}$ and $B^{n \text {,ét }}$ at the stalks, where $\mathcal{H}_{\text {ét }}^{j}\left(\mu_{2^{\nu}}^{\otimes i}\right)\left(\operatorname{resp} . \mathcal{K}_{n}\left(\mathbf{Z} / 2^{\nu}\right)\right)$ is the Zariski sheaf associated to the presheaf $U \mapsto H_{\text {ét }}^{j}\left(U, \mu_{2^{\nu}}^{\otimes i}\right)$ (resp. $U \mapsto$ $K_{n}\left(U, \mathbf{Z} / 2^{\nu}\right)$ ). The homomorphisms of sheaves $\mathcal{B}^{n}$ and $\mathcal{B}^{n \text {,ét }}$ have properties similar to those of proposition 2.1. The same holds when replacing the Zariski topology by the Nisnevich topology of [35].

Proof. It is enough to construct $\mathcal{B}^{n}$ over any affine scheme, hence over any affine scheme $X$ of finite type over $\operatorname{Spec} \mathbf{Z}[1 / 2, i]$ or $\mathbf{F}_{p}$. We proceed exactly as above. If $X$ is smooth over $\operatorname{Spec} \mathbf{Z}[1 / 2, i]$ or $\mathbf{F}_{p}$, the argument in step II) constructs $\mathcal{B}^{n}$ over $X$ and shows that it is contravariant in $X$. In general, we write $X$ as a closed subscheme of $\mathbf{A}_{\mathbf{Z}[1 / 2, i]}^{N}$ or $\mathbf{A}_{\mathbf{F}_{p}}^{N}$ for $N$ large, henselize the latter along $X$ and mimic the argument in step III).

\section{Proofs of theorem 1 , TheOREM 2 AND COROLlary 1}

Proof of theorem 1 a). The construction of section 2 reduces us to the case of a field. We shall use a spectral sequence

$$
E_{2}^{p, q} \Rightarrow K_{-p-q}\left(F, \mathbf{Z} / 2^{\nu}\right)
$$

where

$$
E_{2}^{p, q}= \begin{cases}H^{p-q}\left(F, \mu_{2^{\nu}}^{\otimes(-q)}\right) & \text { if } p, q \leq 0 \\ 0 & \text { otherwise }\end{cases}
$$

Let us recall some facts on this spectral sequence. In [4], Bloch and Lichtenbaum construct a spectral sequence

$$
E_{2}^{p, q}=H^{p-q}(F, \mathbf{Z}(-q)) \Rightarrow K_{-p-q}(F)
$$


where the $E_{2}$-terms are motivic cohomology of $F$, defined as Bloch's higher Chow groups [3] renumbered. In [39], Rognes and Weibel construct a variant with finite coefficients:

$$
E_{2}^{p, q}=H^{p-q}(F, \mathbf{Z} / m(-q)) \Rightarrow K_{-p-q}(F, \mathbf{Z} / m)
$$

where the $E_{2}$-terms are now motivic cohomology with finite coefficients. Now, for $m=2^{\nu}$, there are isomorphisms

$$
H^{p-q}\left(F, \mathbf{Z} / 2^{\nu}(-q)\right)= \begin{cases}H^{p-q}\left(F, \mu_{2^{\nu}}^{\otimes(-q)}\right) & \text { if } p, q \leq 0 \\ 0 & \text { otherwise. }\end{cases}
$$

Indeed, this follows from Voevodsky's proof of the Milnor conjecture [52] and the main result of Geisser-Levine [20]. Before [20], this isomorphism was proven in characteristic 0 by Suslin-Voevodsky [47], modulo the identification of Bloch's motivic cohomology with Voevodsky's motivic cohomology [46].

Then Friedlander and Suslin found a fundamental reinterpretation of the construction in [4], allowing them and Levine to globalise the Bloch-Lichtenbaum spectral sequence to smooth schemes over a field (Friedlander-Suslin, in preparation) and even regular schemes over a regular base of dimension $\leq 1$ (Levine [32]). This also gave another, direct, construction of the Rognes-Weibel variant with finite coefficients. Finally, it allowed Friedlander-Suslin (in preparation) and Levine [33] to provide these spectral sequences with a product structure.

The product structure on (3.1) and the easier existence of transfers allow us to play the same game as in [26] and [28], using the anti-Chern classes to kill all differentials of the spectral sequence and show that the $E_{\infty}$ filtration on the abutment is split by them (see [28, proof of th. 3.1]). Let us give some details on this "game":

1) Consider the composition

$$
H^{0}\left(F, \mu_{2^{\nu}}^{\otimes i}\right) \stackrel{\beta^{i, 0}}{\longrightarrow} K_{2 i}\left(F, \mathbf{Z} / 2^{\nu}\right) \stackrel{e}{\rightarrow} H^{0}\left(F, \mu_{2^{\nu}}^{\otimes i}\right)
$$

where the second map is the edge-homomorphism from the spectral sequence. We claim that this composition is an isomorphism. To see this, we immediately reduce to the case where $F$ is separably closed; then $\beta^{i, 0}$ and $e$ are both isomorphisms (the first one by $[29$, cor. $9.5(\mathrm{v})]$ ). (With a little more effort, one could prove that the composition is actually the identity.)

2) Consider the composition

$$
H^{1}\left(F, \mu_{2^{\nu}}^{\otimes i}\right) \stackrel{\beta^{i, 1}}{\longrightarrow} K_{2 i-1}\left(F, \mathbf{Z} / 2^{\nu}\right) \stackrel{e^{\prime}}{\rightarrow} H^{1}\left(F, \mu_{2^{\nu}}^{\otimes i}\right)
$$

where $e^{\prime}$ is also an edge homomorphism from the spectral sequence. We claim that this composition is again an isomorphism. Using 1), the multiplicativity of the spectral sequence, its compatibility with transfers and proposition 2.2 , we reduce to $i=1$. Then there is a commutative diagram, stemming from the definition of 
$\beta^{1,1}$

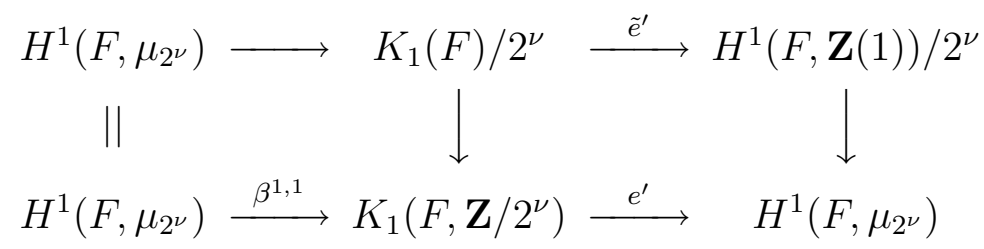

where $\tilde{e}^{\prime}$ stems from an edge homomorphism of the integral spectral sequence. By definition of $\beta^{1,1}$, the top left horizontal map is an isomorphism and so is $\tilde{e}^{\prime}$ by the integral motivic spectral sequence. Since the right vertical map coincides with the Kummer theory isomorphism, the claim is proven.

We have split off one summand from the $K$-groups and also from (3.1). We can then go on, using the next anti-Chern classes, and split off higher and higher chunks from both $K_{*}\left(F, \mathbf{Z} / 2^{\nu}\right)$ and the spectral sequence. More precisely, one shows inductively on $j$ that

- all differentials leaving $E_{r}^{p, q}$ are 0 for $p-q<j$;

- $\operatorname{Im} \beta_{F}^{i, j} \subseteq F^{j-i} K_{2 i-j}\left(F, \mathbf{Z} / 2^{\nu}\right)$, where $F^{*} K_{2 i-j}\left(F, \mathbf{Z} / 2^{\nu}\right)$ is the filtration induced by (3.1);

- The composition

$$
H^{j}\left(F, \mu_{2^{\nu}}^{\otimes i}\right) \stackrel{\beta_{F}^{i, j}}{\longrightarrow} F^{j-i} K_{2 i-j}\left(F, \mathbf{Z} / 2^{\nu}\right) \rightarrow E_{\infty}^{j-i,-i} \hookrightarrow E_{2}^{j-i,-i}=H^{j}\left(F, \mu_{2^{\nu}}^{\otimes i}\right)
$$

is an isomorphism.

Since (3.1) is convergent, we find in the end that it canonically degenerates and that $B_{F}^{n}$ is bijective.

Proof of theorem $\mathbf{1} \mathbf{b}$ ). Note that $(2.1)$ and $(2.2)$ exist for any $A$ (see $[28, \S \S 1$ and 2]). By a), they can now be completed into commutative triangles

$$
\begin{aligned}
& H_{n}(A) \\
& u_{A}^{n} \nearrow \\
& \coprod_{i \leq n} K_{2 i-n}^{M}(n-i)\left(A, \mathbf{Z} / 2^{\nu}\right) \quad B_{A}^{n} \downarrow^{2} \\
& \eta_{A}^{n} \searrow \\
& K_{n}\left(A, \mathbf{Z} / 2^{\nu}\right)
\end{aligned}
$$

where $u_{A}^{n}$ and $\eta_{A}^{n}$ are sums of the $u_{A}^{i, j}$ and the $\eta_{A}^{i, j}$. Theorem $1 \mathrm{~b}$ ) claims that $\eta_{A}^{n}$ is surjective; to see this, it is enough to show that $u_{A}^{n}$ is surjective.

We first show that the Galois symbol $K_{j}^{M}(A) / 2^{\nu} \stackrel{u_{A}^{0, j}}{\longrightarrow} H_{\text {ét }}^{j}\left(A, \mu_{2^{\nu}}^{\otimes j}\right)$ is surjective (cf. [24]). To do this, we proceed as for a) along steps I) - IV) of the proof of proposition 2.1. If $A$ is a field, this is part of Voevodsky's theorem. Suppose $A$ is 
as in step II). We have a commutative diagram of complexes

$$
\begin{aligned}
& 0 \longrightarrow K_{j}^{M}(A) / 2^{\nu} \longrightarrow K_{j}^{M}(E) / 2^{\nu} \longrightarrow \coprod_{x \in X^{(1)}} K_{j-1}^{M}(\kappa(x)) / 2^{\nu} \\
& u_{A}^{0, j} \downarrow \quad u_{E}^{0, j} \downarrow 2 \quad\left(u_{\kappa(x)}^{0, j-1}\right) \downarrow 2 \\
& 0 \longrightarrow H_{\text {êt }}^{j}\left(A, \mu_{2^{\nu}}^{\otimes j}\right) \longrightarrow H_{\text {ét }}^{j}\left(E, \mu_{2^{\nu}}^{\otimes j}\right) \longrightarrow \coprod_{x \in X^{(1)}} H_{\text {ét }}^{j-1}\left(\kappa(x), \mu_{2^{\nu}}^{\otimes j}\right) \text {. }
\end{aligned}
$$

The bottom row is exact by the Bloch-Ogus-Gillet theorem, and the top row is exact at $K_{j}^{M}(E) / 2^{\nu}$ by an unpublished theorem of Gabber [18] (see also [13]). Therefore $u_{A}^{0, j}$ is surjective.

Suppose $A$ is as in step III). Using the same construction as in the proof of a), we get a commutative diagram

$$
\begin{array}{ccc}
K_{j}^{M}(B) / 2^{\nu} & \longrightarrow K_{j}^{M}(A) / 2^{\nu} \\
u_{B}^{0, j} \downarrow & u_{A}^{0, j} \downarrow \\
H_{\text {ét }}^{j}\left(B, \mu_{2^{\nu}}^{\otimes j}\right) & \sim H_{\text {ét }}^{j}\left(A, \mu_{2^{\nu}}^{\otimes j}\right)
\end{array}
$$

in which $u_{B}^{0, j}$ is surjective by step II). Since $K_{j}^{M}(A)$ and $K_{j}^{M}(B)$ are generated by units and $B$ is semi-local, the top horizontal map is surjective and so is $u_{A}^{0, j}$. Finally, step IV) follows from step III) by a passage to the limit, just as in the proof of a).

We now deal with the general case and prove that

$$
K_{j}^{M}(i-j)\left(A, \mathbf{Z} / 2^{\nu}\right) \stackrel{u_{A}^{i-j, j}}{\longrightarrow} H_{\text {ét }}^{j}\left(A, \mu_{2^{\nu}}^{\otimes i}\right)
$$

is surjective for all $A, i, j$. Let $A^{\prime}$ be the smallest finite étale extension of $A$ such that the étale sheaf $\mu_{2^{\nu}}^{\otimes(i-j)}$ is constant over Spec $A^{\prime}$, and let $G=\operatorname{Gal}\left(A^{\prime} / A\right)$. Consider the commutative diagram

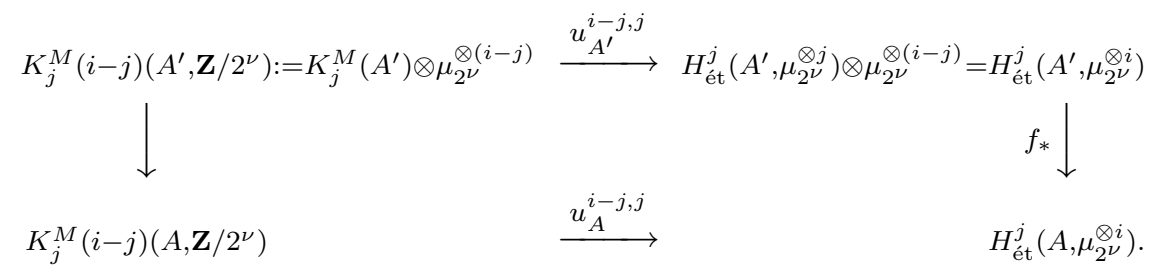

Here the left vertical map is given by the definition of $K_{j}^{M}(i-j)\left(A, \mathbf{Z} / 2^{\nu}\right)=$ $K_{j}^{M}(i-j)\left(A^{\prime}, \mathbf{Z} / 2^{\nu}\right)_{G}$, and the right vertical map is the direct image map associated to the finite morphism $f: \operatorname{Spec} A^{\prime} \rightarrow \operatorname{Spec} A$. By the first part of the proof, $u_{A^{\prime}}^{i-j, j}$ is surjective. By [27, th. 1], the right vertical map in the diagram is surjective, and therefore so is $u_{A}^{i-j, j}$. 
Proof of theorem $1 \mathbf{c}$ ) and $\mathbf{d}$ ). These are special cases of a), in view of the construction of $B_{F}^{n}$.

Corollary 3.1. Let $A$ be as in theorem 1. Then the natural map

$$
K_{n}\left(A, \mathbf{Z} / 2^{\nu}\right) \rightarrow K_{n}\left(A, \mathbf{Z} / 2^{\nu}\right)\left[\beta^{-1}\right]
$$

is a split injection for all $n \geq 0$ and an isomorphism for $n \geq c d_{2}(A)-1$.

Proof. Arguing as in the proof of theorem 1 a), we prove that the homomorphisms $B_{A}^{n \text {,ét }}$ of proposition 2.1 are isomorphisms. Here we reduce to the case where $A$ is a finitely generated field and use Thomason's spectral sequence [51, th. 4.1]; or, more directly, we use the Bott localisation of the motivic spectral sequence. It remains to observe that, in the diagram of proposition 2.1, the left vertical map is a split injection in general and an isomorphism for $n \geq c d_{2}(A)-1$.

Corollary 3.2. If $\mathrm{cd}_{2}(A)<+\infty$, the natural map

$$
K_{n}\left(A, \mathbf{Z} / 2^{\nu}\right)\left[\beta^{-1}\right] \rightarrow K_{n}^{\text {ét }}\left(A, \mathbf{Z} / 2^{\nu}\right)
$$

is an isomorphism for all $n \in \mathbf{Z}$.

Proof. Use the Dwyer-Friedlander spectral sequence of [11, prop. 5.2] (or rather its non-connective analogue) and split it with the help of the $B_{A}^{n \text {,ét }}$ just as in the proof of theorem 1 a).

Corollary 3.3. For all $n, \nu \geq 0$, the homomorphisms of sheaves $\mathcal{B}^{n}$ and $\mathcal{B}^{n \text {,ét }}$ of proposition 2.3 are isomorphisms (for the Zariski or the Nisnevich topology).

Corollary 3.4. a) For any scheme $X$ over $\mathbf{Z}[1 / 2, i]$ or $\mathbf{F}_{p}, p>2$ and any $r, s \geq 0$, there is a natural isomorphism of abelian groups:

$$
\coprod_{i \leq s} H_{\text {Zar }}^{r}\left(X, \mathcal{H}_{\text {ét }}^{2 i-s}\left(\mu_{2^{\nu}}^{\otimes i}\right)\right) \stackrel{\sim}{\longrightarrow} H_{\text {Zar }}^{r}\left(X, \mathcal{K}_{s}\left(\mathbf{Z} / 2^{\nu}\right)\right) .
$$

b) Suppose $X$ is smooth over a field or a Dedekind domain. Then the above isomorphism refines to

$$
\coprod_{r+s \leq 2 i \leq 2 s} H_{\text {Zar }}^{r}\left(X, \mathcal{H}_{\text {ét }}^{2 i-s}\left(\mu_{2^{\nu}}^{\otimes i}\right)\right) \stackrel{\sim}{\rightarrow} H_{\text {Zar }}^{r}\left(X, \mathcal{K}_{s}\left(\mathbf{Z} / 2^{\nu}\right)\right) .
$$

a) and b) hold when replacing $\mathcal{K}_{*}\left(\mathbf{Z} / 2^{\nu}\right)$ by $\mathcal{K}_{*}\left(\mathbf{Z} / 2^{\nu}\right)\left[\beta^{-1}\right]$, and summing over all $i$ in a) and $r+s \leq 2 i$ in b). Finally, the same holds when replacing Zariski cohomology by Nisnevich cohomology.

Proof. This is simply evaluating corollary 3.3 at $X$. b) follows from the fact that $H_{\text {Zar }}^{r}\left(X, \mathcal{H}_{\text {ét }}^{2 i-s}\left(\mu_{2^{\nu}}^{\otimes i}\right)\right)=0$ for $r>2 i-s$ (Gersten's conjecture).

Proposition 3.5. Let $X$ be a Noetherian scheme of finite Krull dimension. Assume that no residue field of $X$ is formally real.

a) If $x \in X$ is a point of codimension $p$, then $c_{2}(x) \leq d_{2}(X)-p$. 
b) If $X \rightarrow S$ is a dominant morphism essentially of finite type, with $d_{2}(S)<\infty$, then $d_{2}(X)=d_{2}(S)+\operatorname{dim}(X / S)$.

Proof. a) follows from [SGA4, exposé X, cor. 2.4]. For b), we reduce to the case where $X \rightarrow S$ is a field extension; then the result follows from [41, p. II-13, prop. $11]$.

Corollary 3.6. For any finite-dimensional Noetherian scheme $X$ over $\operatorname{Spec} \mathbf{Z}[1 / 2, i]$ of $\mathbf{F}_{p}, p>2$, and any $\nu \geq 1$, the natural map

$$
H_{\text {Nis }}^{r}\left(X, \mathcal{K}_{s}\left(\mathbf{Z} / 2^{\nu}\right)\right) \rightarrow H_{\text {Nis }}^{r}\left(X, \mathcal{K}_{s}\left(\mathbf{Z} / 2^{\nu}\right)\left[\beta^{-1}\right]\right)
$$

is split injective for all $r, s$ and bijective for all $r$ and $s \geq d_{2}(X)-1$.

Proof. Using corollary 3.4, it is enough to check that the Nisnevich sheaf $\mathcal{H}^{q}\left(\mu_{2^{\nu}}^{\otimes i}\right)$ is 0 for $q>d_{2}(X)$. Its stalk at a point $x \in X$ is $H_{\text {et }}^{q}\left(\mathcal{O}_{X, x}^{h}, \mu_{2^{\nu}}^{\otimes i}\right)$, where $\mathcal{O}_{X, x}^{h}$ is the henselisation of $\mathcal{O}_{X, x}$. By [SGA4, exposé XII, cor. 5.5], this group coincides with $H_{\text {et }}^{q}\left(\kappa(x), \mu_{2^{\nu}}^{\otimes i}\right)$, which is 0 by proposition 3.5 a) (note that $X$ satisfies the assumptions of this proposition).

Proposition 3.7 (Weibel). Let $X$ be an excellent Noetherian scheme of dimension $\leq 1$. Let $K^{B}(X)$ be Thomason's Bass extension of the $K$-theory spectrum of $X$ [51, def. 6.4]. Then there is a canonical isomorphism $K_{-1}^{B}(X) \simeq H_{\text {êt }}^{1}(X, \mathbf{Z})$.

Proof. ${ }^{2}$ By [51, th. 10.3], for any quasi-compact, quasi-separated $X$, the natural map of spectra

$$
K^{B}(X) \rightarrow \mathbb{H}_{\mathrm{Nis}}\left(X, \mathcal{K}^{B}\right)
$$

is a homotopy equivalence. Here, $\mathbb{H}_{\text {Nis }}\left(X, \mathcal{K}^{B}\right)$ is as in [48, def. 1.33]. Hence a spectral sequence

$$
E_{2}^{p, q}=H_{\text {Nis }}^{p}\left(X, \mathcal{K}_{-q}^{B}\right) \Rightarrow K_{-p-q}^{B}(X)
$$

where $\mathcal{K}_{-q}^{B}$ is the Nisnevich sheaf associated to the presheaf $U \mapsto K_{-q}^{B}(U)$. Since $\operatorname{dim} X \leq 1$, this spectral sequence yields a short exact sequence

$$
0 \rightarrow H_{\mathrm{Nis}}^{1}\left(X, \mathcal{K}_{0}^{B}\right) \rightarrow K_{-1}^{B}(X) \rightarrow H_{\mathrm{Nis}}^{0}\left(X, \mathcal{K}_{-1}^{B}\right) \rightarrow 0 .
$$

The rank identifies canonically $\mathcal{K}_{0}^{B}$ to the constant sheaf $\mathbf{Z}$, and the natural map $H_{\mathrm{Nis}}^{1}(X, \mathbf{Z}) \rightarrow H_{\mathrm{et}}^{1}(X, \mathbf{Z})$ is an isomorphism. It remains to see that $H_{\mathrm{Nis}}^{0}\left(X, \mathcal{K}_{-1}^{B}\right)=$ 0 , i.e. that $K_{-1}^{B}(X)=0$ for $X=\operatorname{Spec} A$ with $A$ local Henselian of dimension $\leq 1$.

By $[54,(1.7 .1)]$, there is an exact sequence

$$
0 \rightarrow L S K_{0}(X) \rightarrow K_{-1}^{B}(X) \rightarrow \operatorname{LPic}(X) \rightarrow 0
$$

and by loc. cit., th. 5.5, $\operatorname{LPic}(X) \simeq H_{\text {êt }}^{1}(X, \mathbf{Z})=0$. Therefore it suffices to show that $L S K_{0}(X)=0$. By [1, th. 7.8], for any 1-dimensional Noetherian ring $A$ whose integral closure is finite over $A$, the determinant map $\tilde{K}_{0}\left(A\left[t, t^{-1}\right]\right) \stackrel{\text { det }}{\longrightarrow} \operatorname{Pic} A\left[t, t^{-1}\right]$

\footnotetext{
${ }^{2}$ I thank Chuck Weibel for his help in this proof.
} 
is an isomorphism, where $\tilde{K}_{0}\left(A\left[t, t^{-1}\right]\right)=\operatorname{Ker}\left(K_{0}\left(A\left[t, t^{-1}\right]\right) \stackrel{r k}{\longrightarrow} H^{0}\left(A\left[t, t^{-1}\right], \mathbf{Z}\right)\right.$. In particular, $L S K_{0}(A):=$ Coker det $=0$. This applies to $A=\Gamma\left(X, \mathcal{O}_{X}\right)$.

Corollary 3.8. For $X$ as in proposition 3.7, the map $K_{0}(X, \mathbf{Z} / n) \rightarrow K_{0}^{B}(X, \mathbf{Z} / n)$ is an isomorphism for any $n>0$.

Proof. By proposition 3.7, $K_{-1}^{B}(X)$ is $n$-torsion-free, as follows from the obvious surjectivity of $H_{\text {ét }}^{0}(X, \mathbf{Z}) \rightarrow H_{\text {ét }}^{0}(X, \mathbf{Z} / n)$. Therefore, the map

$$
K_{0}(X) / n=K_{0}^{B}(X) / n \rightarrow K_{0}^{B}(X, \mathbf{Z} / n)
$$

is bijective.

Proof of theorem 2. The assumption implies that all Hensel local rings of $X$ satisfy the assumptions of theorem 1 .

Smashing the equivalence (3.3) with the Moore spectrum $M\left(\mathbf{Z} / 2^{\nu}\right)$ yields another homotopy equivalence

$$
K^{B}\left(X, \mathbf{Z} / 2^{\nu}\right) \stackrel{\approx}{\rightarrow} \mathbb{H}_{\text {Nis }}^{;}\left(X, \mathcal{K}^{B}\left(\mathbf{Z} / 2^{\nu}\right)\right)
$$

which in turn yields a strongly convergent spectral sequence

$$
E_{2}^{p, q}=H_{\mathrm{Nis}}^{p}\left(X, \mathcal{K}_{-q}^{B}\left(\mathbf{Z} / 2^{\nu}\right)\right) \Rightarrow K_{-p-q}^{B}\left(X, \mathbf{Z} / 2^{\nu}\right) .
$$

This spectral sequence maps to the analogous spectral sequence for Bott-localised $K^{B}$-theory

$$
H_{\mathrm{Nis}}^{p}\left(X, \mathcal{K}_{-q}^{B}\left(\mathbf{Z} / 2^{\nu}\right)\left[\beta^{-1}\right]\right) \Rightarrow K_{-p-q}^{B}\left(X, \mathbf{Z} / 2^{\nu}\right)\left[\beta^{-1}\right]
$$

(compare $[51,(11.8 .1)]$ ). There are natural transformations

$$
K \rightarrow K^{T T} \rightarrow K^{B}
$$

For any quasi-compact quasi-separated $X$, the map $K_{n}^{T T}(X) \rightarrow K_{n}^{B}(X)$ is an isomorphism for $n \geq 0[51$, th. $7.5 \mathrm{a})]$, hence the $\operatorname{map} K_{n}^{T T}\left(X, \mathbf{Z} / 2^{\nu}\right) \rightarrow K_{n}^{B}\left(X, \mathbf{Z} / 2^{\nu}\right)$ is an isomorphism for $n \geq 1$. The map $K_{n}^{T T}\left(X, \mathbf{Z} / 2^{\nu}\right)\left[\beta^{-1}\right] \rightarrow K_{n}^{B}\left(X, \mathbf{Z} / 2^{\nu}\right)\left[\beta^{-1}\right]$ is an isomorphism for all $n[51,(11.4 .2)]$. Also, since $X$ is locally quasi-projective for the Zariski topology, the map of Zariski sheaves $\mathcal{K}_{n}\left(\mathbf{Z} / 2^{\nu}\right) \rightarrow \mathcal{K}_{n}^{T T}\left(\mathbf{Z} / 2^{\nu}\right)$ is an isomorphism for all $n$ [51, prop. 3.10], and the same holds with the Bott element inverted.

In particular, the group $H_{\mathrm{Nis}}^{p}\left(X, \mathcal{K}_{-q}^{B}\left(\mathbf{Z} / 2^{\nu}\right)\right)$ coincides with $H_{\mathrm{Nis}}^{p}\left(X, \mathcal{K}_{-q}\left(\mathbf{Z} / 2^{\nu}\right)\right)$ provided $-q \geq 1$. Hence, by corollary 3.6, the map of spectral sequences is an isomorphism on the $E_{2}^{p, q}$ terms for $-q \geq \max \left(d_{2}(X)-1,1\right)$ and is split injective for all $q \leq-1$. The conclusion follows for Thomason-Trobaugh $K$-theory.

For $K^{\prime}$-theory, we use the Quillen spectral sequence

$$
E_{1}^{p, q}=\coprod_{x \in X^{(p)}} K_{-p-q}\left(\kappa(x), \mathbf{Z} / 2^{\nu}\right) \Rightarrow K_{-p-q}^{\prime}\left(X, \mathbf{Z} / 2^{\nu}\right)
$$

and its analogue for $K^{\prime}$-theory with the Bott element inverted (compare [36, th. 5.5.4]). Here $X^{(p)}$ denotes the set of points of $X$ of codimension $p$. By corollary 
3.1, the natural map between the two spectral sequences is split injective on the $E_{1}$-terms, and bijective on the $E_{1}^{p, q}$-terms provided $-p-q \geq \sup _{x \in X^{(p)}} c d_{2}(x)-1$. By proposition 3.5 a), the right hand side is $\leq d_{2}(X)-p$. Hence the natural map induces a bijection on $E_{1}^{p, q}$ as soon as $-q \geq d_{2}(X)-1$, and the result follows.

Finally, let us prove c). By the non-connective analogue of [11, prop. 5.2], the natural map

$$
K^{\text {ét }}\left(X, \mathbf{Z} / 2^{\nu}\right) \rightarrow \mathbb{H}_{\text {ét }}\left(X, \mathcal{K}^{\text {ét }}\left(\mathbf{Z} / 2^{\nu}\right)\right)
$$

is a weak equivalence. It follows by a simple argument that the natural map

$$
K^{\text {ét }}\left(X, \mathbf{Z} / 2^{\nu}\right) \rightarrow \mathbb{H}_{\text {Nis }}\left(X, \mathcal{K}^{\text {ét }}\left(\mathbf{Z} / 2^{\nu}\right)\right)
$$

is a weak equivalence as well. We then have a commutative diagram

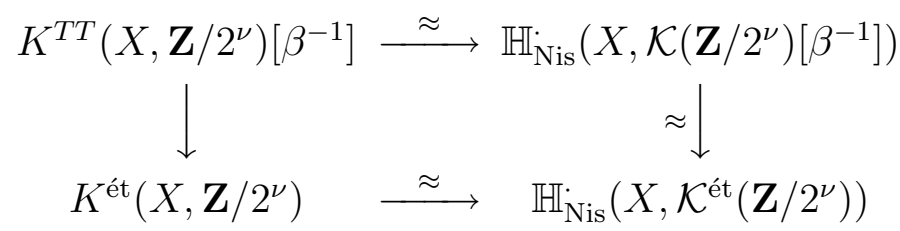

where the top row is a weak equivalence by the considerations at the beginning of the proof (compare $[51,(11.8 .1)]$ ), and the right column is a weak equivalence by corollary 3.2 .

Proof of corollary 1. By proposition $3.5 \mathrm{~b}$ ), we have

$$
d_{2}(X)=\operatorname{dim}(X / S)+d_{2}(S)
$$

and moreover

$$
d_{2}(S)= \begin{cases}2 & \text { in case (i) } \\ 1 & \text { in case (ii) } \\ 0 & \text { in case (iii) } \\ d+1 & \text { in case (iv) }\end{cases}
$$

where, in case (iv), $d$ is the dimension of $k$.

With the exception $\operatorname{dim}(X / S)+d_{2}(S) \leq 2$, the case of $K^{T T}$-theory follows from theorem 2 and the fact that $K_{n}\left(X, \mathbf{Z} / 2^{\nu}\right)\left[\beta^{-1}\right]$ is finite for all $n$, as the abutment of a spectral sequence with $E_{2}$-terms étale cohomology groups [51, th. 11.5] which are finite by the classical finiteness theorems for étale cohomology [10, Th. finitude]. In the exceptional case, we are

1. either in case (i), with $\operatorname{dim}(X / S) \leq 0$;

2. either in case (ii), with $\operatorname{dim}(X / S) \leq 1$;

3. either in case (iii), with $\operatorname{dim}(X / S) \leq 2$;

4. or in case (iv), with $\operatorname{dim} X \leq 1$ (note that $c d_{2}(k) \geq 1$ ).

If $\operatorname{dim} X \leq 1$, by corollary 3.8, the map of spectral sequences in the proof of theorem 2 is an isomorphism even for $q=0$. The assertion then follows as above. 
The only case where $\operatorname{dim} X$ can equal 2 is case (iii). If $X$ is regular, then $K_{i}^{B}(X)=0$ for $i<0$; this completes the proof of corollary 1 for $K^{T T}$-theory.

For $K^{\prime}$-theory, it is enough in view of theorem 2 to show that $K_{n}^{\prime}\left(X, \mathbf{Z} / 2^{\nu}\right)\left[\beta^{-1}\right]$ is finite for all $n \in \mathbf{Z}$. This is true for $X$ regular, by reduction to $K$-theory. In general, we can apply [EGA4, cor. 6.12.6] in each case of the corollary to find in $X$ a regular dense open subset $U$. Using the localisation exact sequence for $X, U$ and $X \backslash U$, we get the result by Noetherian induction.

\section{Higher Chern Classes with values in truncated Étale COHOMOLOGY}

4.1. Construction. Let $S=\operatorname{Spec} R$, where $R$ is a field or a Dedekind domain. Suppose 2 is invertible on $S$. Using Gillet's method [21], we construct higher Chern classes

$$
c_{i, j}: K_{2 i-j}\left(X, \mathbf{Z} / 2^{\nu}\right) \rightarrow H_{\mathrm{Zar}}^{j}\left(X, B / 2^{\nu}(i)\right)
$$

for any $S$-scheme $X$ and $2 i-j \geq 2$. Here $B / 2^{\nu}(i)=\tau_{\leq i} R \alpha_{*} \mu_{2^{\nu}}^{\otimes i}$, where $\alpha$ is the projection of the big étale site of $S$ onto its big Zariski site, as in [47].

A) Looking at the arguments in $[21, \S 2]$, one sees that to get Chern classes

$$
C_{i} \in H_{\mathrm{Zar}}^{2 i}\left(X, \mathcal{G} \mathcal{L}\left(\mathcal{O}_{X}\right), B / 2^{\nu}(i)\right)
$$

$[21$, p. 225] for any $S$-scheme $X$, it is enough to prove a projective bundle theorem for vector bundles over $X$, where $X$ is (essentially) smooth over $S$. (The other axioms of [21, def. 1.1 and 1.2], and in particular the existence of a Borel-Moore homology theory, are not needed.) For such a vector bundle $E$ of rank $r$, with associated projective bundle $\mathbb{P}(E) \stackrel{\pi}{\rightarrow} X$, the projective bundle formula will take the form

$$
\coprod_{i=0}^{r} B / 2^{\nu}(n-i)_{X}[-2 i] \stackrel{\sim}{\longrightarrow} R \pi_{*} B / 2^{\nu}(n)_{\mathbb{P}(E)}
$$

for all $n \geq 0$, in the derived category of Zariski sheaves over $X$, where the $i$-th component of the isomorphism is given by pull-back under $\pi$ followed by cupproduct by the $i$-th power of a certain class $\xi \in H^{2}\left(\mathbb{P}(E), B / 2^{\nu}(1)\right)$.

We first construct the class $\xi$. The Kummer exact sequence in the étale topology

$$
1 \rightarrow \mu_{2^{\nu}} \rightarrow \mathbb{G}_{m} \stackrel{2^{\nu}}{\rightarrow} \mathbb{G}_{m} \rightarrow 1
$$

yields, by higher direct image and truncation, a morphism in the derived category

$$
\tau_{\leq 1} R \alpha_{*} \mathbb{G}_{m} \rightarrow B / 2^{\nu}(1)[1]
$$

hence a map in cohomology

$$
H_{\text {Zar }}^{1}\left(\mathbb{P}(E), \tau_{\leq 1} R \alpha_{*} \mathbb{G}_{m}\right) \rightarrow H_{\text {Zar }}^{2}\left(\mathbb{P}(E), B / 2^{\nu}(1)\right) .
$$


But $H_{\text {Zar }}^{1}\left(\mathbb{P}(E), \tau_{\leq 1} R \alpha_{*} \mathbb{G}_{m}\right)=H_{\text {Zar }}^{1}\left(\mathbb{P}(E), R \alpha_{*} \mathbb{G}_{m}\right)=H_{\text {ét }}^{1}\left(\mathbb{P}(E), \mathbb{G}_{m}\right)=\operatorname{Pic} \mathbb{P}(E)$. The class $\xi$ is the image by (4.4) of the class of the tautological bundle $\mathcal{O}(1)$ in $\operatorname{Pic} \mathbb{P}(E)$.

We note that the morphism (4.3) induces, for all $q \in[0, n]$, a morphism of truncated complexes

$$
\coprod_{i=0}^{r}\left(\tau_{\leq q-i} B / 2^{\nu}(n-i)_{X}\right)[-2 i] \rightarrow R \pi_{*} \tau_{\leq q} B / 2^{\nu}(n)_{\mathbb{P}(E)} .
$$

Since the corresponding filtration on the left hand side of (4.3) is exhaustive, in order to prove that (4.3) is an isomorphism it suffices to prove that it induces an isomorphism on the successive cones of (4.5), that is, on

$$
\coprod_{i=0}^{r} R^{q-i} \alpha_{*} \mu_{2^{\nu}}^{\otimes(n-i)}[-q-i] \rightarrow R \pi_{*} R^{q} \alpha_{*} \mu_{2^{\nu}}^{\otimes n}[-q]
$$

or on

$$
\coprod_{i=0}^{r} R^{q-i} \alpha_{*} \mu_{2^{\nu}}^{\otimes(n-i)}[-i] \rightarrow R \pi_{*} R^{q} \alpha_{*} \mu_{2^{\nu}}^{\otimes n} .
$$

Note that the morphism in (4.6) is induced by the powers of the image of $\xi$ in $H_{\text {Zar }}^{1}\left(\mathbb{P}(E), R^{1} \alpha_{*} \mu_{2^{\nu}}\right)$.

We now want to mimick the proof of [21, lemma 8.11]. For this, it is convenient to introduce cycle homology groups as in [40]. Recall that, for any $i \in \mathbf{Z}$, the functor on fields

$$
k \mapsto\left(H^{q}\left(k, \mu_{2^{\nu}}^{\otimes(q+i)}\right)\right)_{q \geq 0}
$$

is a cycle module over $\mathbf{Z}[1 / 2]$ in the sense of Rost (loc. cit., remark 2.5). For any scheme $X$, we define

$$
A_{p}\left(X, H^{q}\left(\mu_{2^{\nu}}^{\otimes n}\right)\right)
$$

as the $p$-th homology group of the complex

$$
\cdots \rightarrow \coprod_{x \in X_{(p)}} H^{p+q}\left(k(x), \mu_{2^{\nu}}^{\otimes(p+n)}\right) \rightarrow \ldots
$$

defined by Kato [31]. Here $X_{(p)}$ denotes the set of points of dimension $p$ in $X$. If $X$ is equidimensional of dimension $d$, we set

$$
A^{p}\left(X, H^{q}\left(\mu_{2^{\nu}}^{\otimes n}\right)\right)=A_{d-p}\left(X, H^{d+q}\left(\mu_{2^{\nu}}^{\otimes d+n}\right)\right)
$$

and extend this definition by additivity if $X$ is a disjoint union of equidimensional schemes.

If $X$ is smooth over a field (resp. a dvr), the Bloch-Ogus theorem (resp. the Bloch-Ogus-Gillet theorem of [22]) gives an isomorphism

$$
A^{p}\left(X, H^{q}\left(\mu_{2^{\nu}}^{\otimes n}\right)\right) \simeq H_{\mathrm{Zar}}^{p}\left(X, R^{q} \alpha_{*} \mu_{2^{\nu}}^{\otimes n}\right) .
$$


The interest of the cycle homology groups is that they tautologically satisfy a localisation theorem: if $Z$ is a closed subset of $X$, with complementary open set $U$, there are long exact sequences [40, p. 356]

$$
\begin{aligned}
\cdots \rightarrow A_{p}\left(Z, H^{q}\left(\mu_{2^{\nu}}^{\otimes n}\right)\right) \rightarrow A_{p}\left(X, H^{q}\left(\mu_{2^{\nu}}^{\otimes n}\right)\right) & \rightarrow A_{p}\left(U, H^{q}\left(\mu_{2^{\nu}}^{\otimes n}\right)\right) \\
& \rightarrow A_{p-1}\left(Z, H^{q}\left(\mu_{2^{\nu}}^{\otimes n}\right)\right) \rightarrow \ldots
\end{aligned}
$$

In particular, if $X$ and $Z$ are regular and $Z$ is purely of codimension $c$ in $X$, we get Gysin exact sequences

$$
\begin{aligned}
\cdots \rightarrow A^{p-c}\left(Z, H^{q-c}\left(\mu_{2^{\nu}}^{\otimes(n-c)}\right)\right) \rightarrow A^{p}\left(X, H^{q}\left(\mu_{2^{\nu}}^{\otimes n}\right)\right) & \rightarrow A^{p}\left(U, H^{q}\left(\mu_{2^{\nu}}^{\otimes n}\right)\right) \\
& \rightarrow A^{p-c+1}\left(Z, H^{q-c}\left(\mu_{2^{\nu}}^{\otimes(n-c)}\right)\right) \rightarrow \ldots
\end{aligned}
$$

This allows us to use Noetherian induction. For example

Lemma 4.1. For any Noetherian scheme $X$ of finite Krull dimension and any $r \geq 0$, the map $[\mathcal{O}, f, r]$ of $[40,3.5 .3]$ :

$$
A_{p}\left(X, H^{q}\left(\mu_{2^{\nu}}^{\otimes n}\right)\right) \rightarrow A_{p+r}\left(\mathbb{A}_{X}^{r}, H^{q+r}\left(\mu_{2^{\nu}}^{\otimes(n+r)}\right)\right)
$$

is an isomorphism, where $\mathcal{O}=\mathcal{O}_{\mathbb{A}_{X}^{r}}$ and $f$ is the projection $\mathbb{A}_{X}^{r} \rightarrow X$.

Proof. We do as in [21, proof of th. 8.3], with a slight simplification. By [40, prop. $4.1(2)$ ], we first reduce to $r=1$. As in [21], we then reduce by Noetherian induction to the case where $X$ is the spectrum of a field $F$. We now have a commutative diagram

$$
\begin{aligned}
0 \rightarrow A^{1}\left(\mathbb{A}_{F}^{1}, H^{q-1}\left(\mu_{2^{\nu}}^{\otimes n}\right)\right) \longrightarrow & H_{\text {ét }}^{q}\left(\mathbb{A}_{F}^{1}, \mu_{2^{\nu}}^{\otimes n}\right) \longrightarrow \\
\imath \uparrow & A^{0}\left(\mathbb{A}_{F}^{1}, H^{q}\left(\mu_{2^{\nu}}^{\otimes n}\right)\right) \rightarrow 0 \\
& H_{\text {ét }}^{q}\left(F, \mu_{2^{\nu}}^{\otimes n}\right) .
\end{aligned}
$$

Here the top row is exact by the coniveau spectral sequence (see below), while the vertical map is an isomorphism by homotopy invariance. On the other hand, the diagonal map is split injective, by (4.7) and the choice of a rational point of $\mathbb{A}_{F}^{1}$. It follows that it is bijective and that $A^{1}\left(\mathbb{A}_{F}^{1}, H^{q-1}\left(\mu_{2^{\nu}}^{\otimes n}\right)\right)=0$.

To proceed, we need products

$$
A^{p}\left(Y, H^{q}\left(\mu_{2^{\nu}}^{\otimes n}\right)\right) \otimes H_{\mathrm{Zar}}^{p^{\prime}}\left(Y, R^{q^{\prime}} \alpha_{*} \mu_{2^{\nu}}^{\otimes n^{\prime}}\right) \rightarrow A^{p+p^{\prime}}\left(Y, H^{q+q^{\prime}}\left(\mu_{2^{\nu}}^{\otimes\left(n+n^{\prime}\right)}\right)\right)
$$

for $Y$ regular over $R$. Such products are constructed in $[40, \S 14]$ when $R$ is a field, in view of Gersten's conjecture. When $R$ is a dvr, the only way we found to construct them is to mimick the arguments of [21, p. 281] and use Gabber's (unpublished) absolute cohomological purity theorem. More precisely, for any equidimensional Noetherian scheme $Y$ of finite Krull dimension, we have a coniveau spectral sequence $[8, \S 1]$

$$
E_{1}^{p, q}(n)=\coprod_{x \in Y^{(p)}} H_{x}^{p+q}\left(Y, \mu_{2^{\nu}}^{\otimes n}\right) \Rightarrow H_{\text {ét }}^{p+q}\left(Y, \mu_{2^{\nu}}^{\otimes n}\right) .
$$


The construction of this spectral sequence shows that there are products

$$
E_{r}^{p, q}(n) \otimes H_{\text {ét }}^{s}\left(Y, \mu_{2^{\nu}}^{\otimes t}\right) \rightarrow E_{r}^{p, q+s}(n+t)
$$

cf. [21, p. 276]. Sheafifying these pairings for $r=1$ yields pairings of sheaves, hence on hypercohomology:

$$
E_{2}^{p, q}(n) \otimes H_{\mathrm{Zar}}^{p^{\prime}}\left(Y, R^{q^{\prime}} \alpha_{*} \mu_{2^{\nu}}^{\otimes n^{\prime}}\right) \rightarrow E_{2}^{p+p^{\prime}, q+q^{\prime}}\left(n+n^{\prime}\right) .
$$

Now, using Gabber's theorem

$$
H_{Z}^{p}\left(X, \mu_{n}^{\otimes i}\right) \simeq H^{p-2 c}\left(Z, \mu_{n}^{\otimes(i-c)}\right)
$$

for a regular pair $(X, Z)$ of codimension $c$, we can identify $E_{2}^{p, q}(n)$ with $A^{p}\left(Y, H^{q}\left(\mu_{2^{\nu}}^{\otimes n}\right)\right)$ when $Y$ is regular (use [EGA4, cor. 6.12.6] again to note that any closed integral subscheme of $Y$ has a dense open regular subset). This gives the desired products.

For any regular scheme $Y$, we have the Leray spectral sequence

$$
E_{2}^{p, q}=H_{\mathrm{Zar}}^{p}\left(Y, R^{q} \alpha_{*} \mu_{2^{\nu}}\right) \Rightarrow H_{\text {ét }}^{p+q}\left(Y, \mu_{2^{\nu}}\right) .
$$

In this spectral sequence, $E_{2}^{2,0}=H_{\mathrm{Zar}}^{2}\left(Y, \mu_{2^{\nu}}\right)=0$, hence there is a canonical morphism

$$
H_{\text {ét }}^{2}\left(Y, \mu_{2^{\nu}}\right) \rightarrow H_{\text {Zar }}^{1}\left(Y, \mathcal{H}_{\text {ét }}^{1}\left(\mu_{2^{\nu}}\right)\right)
$$

hence any line bundle on $Y$ has a class in the left hand side. For $X$ regular and $E$ a vector bundle of rank $r$, we define homomorphisms

$$
\coprod_{i=0}^{r} A^{p-i}\left(X, H^{q-i}\left(\mu_{2^{\nu}}^{\otimes(n-i)}\right)\right) \rightarrow A^{p}\left(\mathbb{P}(E), H^{q}\left(\mu_{2^{\nu}}^{\otimes n}\right)\right)
$$

by cupping with the powers of the class $\xi \in H_{\text {Zar }}^{1}\left(\mathbb{P}(E), \mathcal{H}_{\text {ét }}^{1}\left(\mu_{2^{\nu}}\right)\right)$ of $\mathcal{O}(1)$. When $X$ is smooth over a field or a dvr, these homomorphisms are compatible with those of (4.6) via (4.7). The fact that (4.6) is an isomorphism will therefore follow from

Proposition 4.2. For any regular $X$ of finite Krull dimension and any $(p, q, n, \nu)$, (4.9) is an isomorphism.

Proof. This is a variant of the proof of [21, lemma 8.11]. Using Noetherian induction (this time with the help of (4.8)), we again reduce to the case where $X$ is the spectrum of a field $F$. Then the vector bundle $E$ is trivial. We can now finish as in loc. cit., using lemma 4.1 to argue by induction on $r$ (see also [14, prop. 3.7]).

B) To define the $c_{i, j}$, we could proceed directly along the lines of [21], using the Hurewicz homomorphism with finite coefficients as in [42]; however, for our purposes, we are forced to do something slightly more complicated.

As in [21, p. 225], we can interpret (4.2) as a map of simplicial sheaves over $X_{\text {Zar }}$

$$
C_{i}: B \mathcal{G} \mathcal{L}\left(\mathcal{O}_{X}\right) \rightarrow \mathcal{K}\left(B / 2^{\nu}(i), 2 i\right)
$$


where the right hand side is a sheaf of Eilenberg-Mac Lane spaces. Applying the functor $\mathbf{Z}_{\infty}$ of Bousfield-Kan, noting that up to homotopy this transforms the left hand side into $\Omega B \mathcal{Q P}\left(\mathcal{O}_{X}\right)$ (the sheaf associated to Quillen's $Q$-construction) and does not change the right hand side, we get, up to zig-zags of weak equivalences, a new map of simplicial sheaves, that we still denote by $C_{i}$ :

$$
C_{i}: \Omega B \mathcal{Q P}\left(\mathcal{O}_{X}\right) \rightarrow \mathcal{K}\left(B / 2^{\nu}(i), 2 i\right)
$$

For any $k \geq 2$, let $P^{k}\left(2^{\nu}\right)$ be a Moore space of level $k$ (denoted by $Y^{k}$ in [42, p. $259]$ ), and let $M\left(2^{\nu}\right)$ be the Moore spectrum for $\mathbf{Z} / 2^{\nu}$ : we have $\Sigma P^{k}\left(2^{\nu}\right)=P^{k+1}\left(2^{\nu}\right)$ and $M\left(2^{\nu}\right)=\Sigma^{\infty-k} P^{k}\left(2^{\nu}\right)$ for any $k \geq 2$. We shall show that (4.10) refines into a collection of maps, for $2 \leq k \leq 2 i$ :

$$
C_{i}^{(k)}: \operatorname{Hom}\left(P^{k}\left(2^{\nu}\right), \Omega B \mathcal{Q P}\left(\mathcal{O}_{X}\right)\right) \rightarrow \mathcal{K}\left(B / 2^{\nu}(i), 2 i-k\right) .
$$

For this, let $H(C)$ denote the Eilenberg-Mac Lane spectrum associated to a complex of abelian groups $C$; for $C=\mathbf{Z} / m[0]$, let us abbreviate this by $H(m)$. If $\mathcal{C}$ is a complex of sheaves of abelian groups over $X_{\text {Zar }}$, we denote by $\mathcal{H}(\mathcal{C})$ the corresponding sheaf of Eilenberg-Mac Lane spectra. We note that there is a canonical weak equivalence

$$
H(\mathbf{Z}) \wedge M\left(2^{\nu}\right) \simeq H\left(2^{\nu}\right) .
$$

From the unit morphism $\Sigma^{\infty} \rightarrow H(\mathbf{Z})$ and this equivalence, we therefore deduce a morphism $\left(\bmod 2^{\nu}\right.$ Hurewicz morphism):

$$
M\left(2^{\nu}\right) \stackrel{h u}{\longrightarrow} H\left(2^{\nu}\right) .
$$

Let $\mathcal{I}$ be an injective $\mathbf{Z} / 2^{\nu}$-resolution of $B / 2^{\nu}(i)$. Denote by $\mathcal{X}$ the simplicial sheaf $\Omega B \mathcal{Q P}\left(\mathcal{O}_{X}\right)$. We can interpret $C_{i}$ as a homotopy class of maps

$$
C_{i}: C_{*}(\mathcal{X}) \otimes \mathbf{Z} / 2^{\nu} \rightarrow \mathcal{I}[2 i]
$$

where $C_{*}$ denotes the standard chain complex. We now have a chain of maps, for any $k \in[2,2 i]$ :

$$
\begin{aligned}
{\left[C_{*}(\mathcal{X}) \otimes \mathbf{Z} / 2^{\nu}, \mathcal{I}[2 i]\right] \simeq\left\{\mathcal{X} \wedge H\left(2^{\nu}\right), \mathcal{H}(\mathcal{I}[2 i])\right\} } \\
\stackrel{h u^{*}}{\longrightarrow}\left\{\mathcal{X} \wedge M\left(2^{\nu}\right), \mathcal{H}(\mathcal{I}[2 i])\right\} \stackrel{\delta}{\rightarrow}\left\{\operatorname{Hom}\left(M\left(2^{\nu}\right), \Sigma^{\infty} \mathcal{X}\right), \mathcal{H}(\mathcal{I}[2 i])\right\} \\
\simeq\left\{\Sigma^{\infty-k} \operatorname{Hom}\left(P^{k}\left(2^{\nu}\right), \Omega^{\infty} \Sigma^{\infty} \mathcal{X}\right), \mathcal{H}(\mathcal{I}[2 i])\right\} \\
\simeq\left[\operatorname{Hom}\left(P^{k}\left(2^{\nu}\right), \Omega^{\infty} \Sigma^{\infty} \mathcal{X}\right), \mathcal{K}\left(B / 2^{\nu}(i), 2 i-k\right)\right] \\
\quad \stackrel{\theta}{\rightarrow}\left[\operatorname{Hom}\left(P^{k}\left(2^{\nu}\right), \mathcal{X}\right), \mathcal{K}\left(B / 2^{\nu}(i), 2 i-k\right)\right] .
\end{aligned}
$$

Here $h u^{*}$ is induced by the mod $2^{\nu}$ Hurewicz homomorphism defined above, $\delta$ (an isomorphism) comes from the self-S-duality of $M\left(2^{\nu}\right)$ and $\theta$ is induced by the stabilisation map $\mathcal{X} \rightarrow \Omega^{\infty} \Sigma^{\infty} \mathcal{X}$. The map $C_{i}^{(k)}$ is the image of $C_{i}$ under this chain. 
For $2<k \leq 2 i$, there are tautological isomorphisms

$$
\begin{aligned}
& \operatorname{Hom}\left(P^{k}\left(2^{\nu}\right), \mathcal{X}\right) \stackrel{\sim}{\longrightarrow} \operatorname{\Omega Hom}\left(P^{k-1}\left(2^{\nu}\right), \mathcal{X}\right) \\
& \mathcal{K}\left(B / 2^{\nu}(i), 2 i-k\right) \stackrel{\sim}{\longrightarrow} \Omega \mathcal{K}\left(B / 2^{\nu}(i), 2 i-k+1\right)
\end{aligned}
$$

and it is clear, by construction, that the diagram

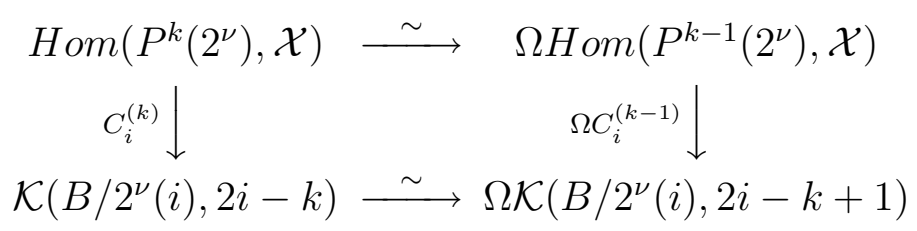

commutes.

Taking homotopy groups of global sections, we get composite maps

$$
\begin{aligned}
c_{i, j}^{(k)}: \pi_{j}\left(\operatorname{Hom}\left(P^{k}\left(2^{\nu}\right), \Omega B \mathcal{Q P}(X)\right)\right) & \\
& \rightarrow \pi_{j}\left(\mathbb{H}_{\text {Zar }}^{\cdot(X, H o m}\left(P^{k}\left(2^{\nu}\right), \Omega B \mathcal{Q P}\left(\mathcal{O}_{X}\right)\right)\right) \\
& \rightarrow \pi_{j}\left(\mathbb{H}_{\text {Zar }}\left(X, \mathcal{K}\left(B / 2^{\nu}(i), 2 i-k\right)\right)\right)
\end{aligned}
$$

or

$$
c_{i, j}^{(k)}: K_{j+k}\left(X, \mathbf{Z} / 2^{\nu}\right) \rightarrow H_{\mathrm{Zar}}^{2 i-j-k}\left(X, B / 2^{\nu}(i)\right)
$$

for $k \geq 2$ and $j+k \leq 2 i$. Here we denote by $\mathbb{H}_{\text {Zar }}(X, \mathcal{Y})$ the global sections of a functorial simplicial cofibrant or flasque resolution of a simplicial sheaf $\mathcal{Y}$, lifting the functor $R \Gamma(X, \mathcal{Y})$ of [7]. The most convenient is to use the Godement resolution of $\mathcal{Y}$, as in [48, def. 1.33], where this is done for a (pre)sheaf of fibrant spectra. Diagram (4.12) shows that

$$
c_{i, j}^{(k)}=c_{i, j+1}^{(k-1)} \quad \text { for } j+k \leq 2 i \text { and } 2<k \leq 2 i .
$$

Definition 4.3. We denote by $c_{i, j}: K_{2 i-j}\left(X, \mathbf{Z} / 2^{\nu}\right) \rightarrow H_{\mathrm{Zar}}^{j}\left(X, B / 2^{\nu}(i)\right)$ the homomorphism $c_{i, l}^{(k)}$ for any pair $(l, k)$ such that $l+k=2 i-j$ and $2 \leq k \leq 2 i$.

This homomorphism is therefore defined only for $2 i-j \geq 2$. For $2 i-j=1$, Gillet's Chern classes from integral $K$-theory exist and are group homomorphisms; they therefore induce homomorphisms

$$
c_{i, 2 i-1}: K_{1}(X) / 2^{\nu} \rightarrow H_{\mathrm{Zar}}^{2 i-1}\left(X, B / 2^{\nu}(i)\right) .
$$

Finally, for $2 i-j=0$, the Chern classes defined in A) induce non-additive maps

$$
c_{i}: K_{0}(X) \rightarrow H_{\text {Zar }}^{2 i}\left(X, B / 2^{\nu}(i)\right) .
$$

It is not true in general that the $c_{i}$ factor through $K_{0}(X) / 2^{\nu}$ (compare [42, II.2.3]). For example, if $k$ is a field, $X=\mathbb{P}_{k}^{n}$ and $x=[\mathcal{O}(1)] \in K_{0}(X)$, then $c_{2^{\nu}}\left(2^{\nu} x\right)=c_{1}(x)^{2^{\nu}}$, which is nonzero for $n>2^{\nu}$. Using Newton polynomials in the $c_{i}$ rather than the $c_{i}$ themselves, we get however homomorphisms $s_{i}: K_{0}(X) / 2^{\nu} \rightarrow$ $H_{\mathrm{Zar}}^{2 i}\left(X, B / 2^{\nu}(i)\right)$. Note that, if $X$ is smooth over a field, the latter group coincides with $C H^{i}(X) / 2^{\nu}$ by the Bloch-Ogus theorem. 
4.2. Relationship with Soulé's étale Chern classes. Pushing truncated étale cohomology to étale cohomology, we get composite classes

$$
c_{i, j}^{\text {ét }}: K_{2 i-j}\left(X, \mathbf{Z} / 2^{\nu}\right) \rightarrow H_{\mathrm{Zar}}^{j}\left(X, B / 2^{\nu}(i)\right) \rightarrow H_{\text {ét }}^{j}\left(X, \mu_{2^{\nu}}^{\otimes i}\right) .
$$

Lemma 4.4. If $X$ is affine, $c_{i, j}^{\text {et }}$ coincide with Soulé's étale Chern class $\bar{c}_{i, j}[42$, II.2.3].

Proof. Soulé's Chern classes are defined via the mod $2^{\nu}$ Hurewicz homomorphisms

$$
K_{i}\left(A, \mathbf{Z} / 2^{\nu}\right) \rightarrow H_{i}\left(G L(A), \mathbf{Z} / 2^{\nu}\right)
$$

which are themselves defined by means of the canonical generators of $H_{i-1}\left(P^{i}\left(2^{\nu}\right), \mathbf{Z} / 2^{\nu}\right)$. Our version of the $\bmod 2^{\nu}$ Hurewicz homomorphisms rather uses the canonical generators of $H^{i-1}\left(P^{i}\left(2^{\nu}\right), \mathbf{Z} / 2^{\nu}\right)$ and $S$-duality. It is easy to check that these two definitions are compatible.

If $X=\operatorname{Spec} A$ for $A$ a local ring, the group $H^{i}\left(X_{\mathrm{Zar}}, B / 2^{\nu}(n)\right)$ reduces to

- $H_{\text {ét }}^{i}\left(X, \mu_{2^{\nu}}^{\otimes n}\right)$ for $i \leq n$

- 0 for $i>n$.

Lemma 4.5. Let $A$ be a local algebra over $\mathbf{Z}[1 / 2]$, and $B / A$ a finite étale extension. Then, for all $i, j$,

$$
i c_{i, j} \circ f_{*}=i f_{*} \circ c_{i, j}
$$

where $f$ is the morphism $\operatorname{Spec} B \rightarrow \operatorname{Spec} A$.

Proof. We proceed in 4 steps, as usual:

I) A is a field. We may assume B is a field as well. The claim follows from lemma 4.4 and [42, proof of th. $2 \mathrm{ii})]$.

II) $\mathrm{A}$ is a local ring of a scheme smooth over a Dedekind domain. We reduce to I) by Gillet's Bloch-Ogus theorem, which implies that the étale cohomology of $A$ injects into that of its field of fractions.

III) $\mathrm{A}$ is a local ring of a scheme of finite type over a Dedekind domain. We use Hoobler's henselian couple trick [24], noting that étale coverings of Spec $A$ lift, plus Gabber's rigidity result [16].

IV) The general case. Passage to the limit.

\section{Proof of theorem 3}

5.1. Effect of the Chern classes on hypercohomology. We now examine the effect of applying total global sections to (4.11) in more detail. For any sheaf $\mathcal{Y}$ of simplicial sets on $X$, there is by [7, th. 3 and remark p. 285] a "fringed" spectral sequence

$$
E_{2}^{p, q}=H_{\text {Zar }}^{p}\left(X, \pi_{-q}(\mathcal{Y})\right) \Rightarrow \pi_{-p-q}\left(\mathbb{H}_{\text {Zar }}(X, \mathcal{Y})\right)
$$

with fringe effect concentrated on the line $p+q=0$. 
By functoriality of $\mathbb{H}^{\cdot}$, any morphism of simplicial sheaves induces a corresponding morphism of spectral sequences. Applying this to $C_{i}^{(k)}$, we get a morphism from the spectral sequence

$$
A_{2}^{p, q}=H_{\text {Zar }}^{p}\left(X, \mathcal{K}_{k-q}\left(\mathbf{Z} / 2^{\nu}\right)\right) \Rightarrow \pi_{-p-q}\left(\mathbb{H}_{\text {Zar }}\left(X, \Omega B \mathcal{Q P}\left(\mathcal{O}_{X}\right)\right)\right)
$$

to the spectral sequence

$$
B_{2}^{p, q}(i)=H_{\mathrm{Zar}}^{p}\left(X, \mathcal{H}^{2 i+q-k}\left(B / 2^{\nu}(i)\right)\right) \Rightarrow H_{\mathrm{Zar}}^{2 i+p+q-k}\left(X, B / 2^{\nu}(i)\right) .
$$

The corresponding morphism $A_{2}^{p, q} \rightarrow B_{2}^{p, q}$ is $H_{\mathrm{Zar}}^{p}\left(X, c_{i,-q}^{(k)}\right)=H_{\mathrm{Zar}}^{p}\left(X, c_{i, 2 i+q-k}\right)$ (definition 4.3).

For both spectral sequences, the simplicial sheaf in question is an infinite loop space. One could therefore use the technique of $[7, \S 3$, remark 1] to deloop them and get rid of the fringe effect. However, $C_{i}^{(k)}$ is not an infinite loop map, hence does not yield a morphism of the corresponding delooped spectral sequences. In other words, we cannot get rid of the fringe effects as long as we work with the $C_{i}^{(k)}$.

Lemma 5.1. Assume $X$ quasi-compact and quasi-separated. Then

a) The Chern classes of definition 4.3 extend to Chern classes from ThomasonTrobaugh $K$-theory

$$
c_{i, j}: K_{2 i-j}^{T T}\left(X, \mathbf{Z} / 2^{\nu}\right) \rightarrow H_{\text {Zar }}^{j}\left(X, B / 2^{\nu}(i)\right) .
$$

b) The spectral sequence (5.1) "abuts" to $K_{k-p-q}^{T T}\left(X, \mathbf{Z} / 2^{\nu}\right)$ (with fringe effect, of course).

Proof. Consider the morphism of simplicial sheaves

$$
\Omega B \mathcal{G} \mathcal{Q}\left(\mathcal{O}_{X}\right) \rightarrow \Omega^{\infty} \mathcal{K}^{T T}\left(\mathcal{O}_{X}\right) \stackrel{\approx}{\rightarrow} \Omega^{\infty} \mathcal{K}^{B}\left(\mathcal{O}_{X}\right)
$$

stemming from (3.5). Note that the right map is a weak equivalence by $[51$, th. 7.5 a)]. Taking total global sections, we get a commutative diagram of simplicial sets

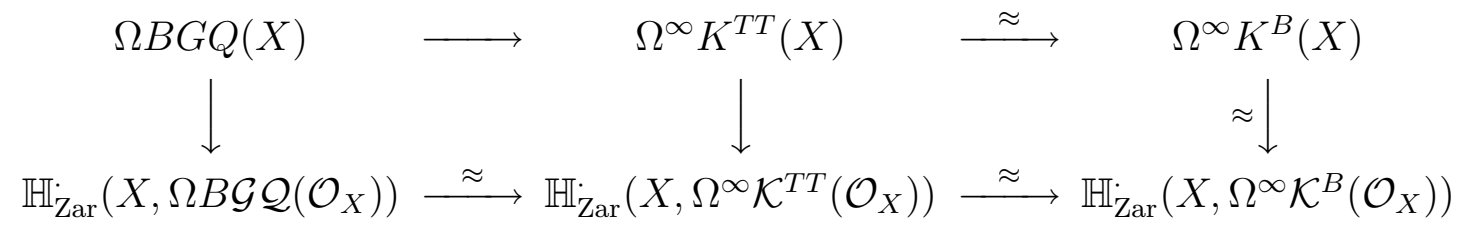

where the right vertical map is a weak equivalence by the Thomason-Trobaugh descent theorem [51, th. 10.3] (or rather its analogue for the Zariski topology) and the fact that homotopy limits commute with desuspension (compare [48, (5.3)]), the top and bottom right horizontal maps are weak equivalences again by $[51$, th. $7.5 \mathrm{a})]$ and the bottom left horizontal map is a weak equivalence as well by [51, prop. 3.10]. Hence there is a weak equivalence

$$
\Omega^{\infty} K^{T T}(X) \approx \mathbb{H}_{\mathrm{Zar}}\left(X, \Omega B \mathcal{G} \mathcal{Q}\left(\mathcal{O}_{X}\right)\right)
$$


and a weak equivalence

$$
\operatorname{Hom}\left(P^{k}\left(2^{\nu}\right), \Omega^{\infty} K^{T T}(X)\right) \approx \mathbb{H}_{\mathrm{Zar}}\left(X, \operatorname{Hom}\left(P^{k}\left(2^{\nu}\right), \Omega B \mathcal{G} \mathcal{Q}\left(\mathcal{O}_{X}\right)\right)\right)
$$

for $k \geq 2$.

\subsection{Proof of theorem 3.}

Proposition 5.2. Let $A$ be a local algebra over $\mathbf{Z}[1 / 2, \sqrt{-1}]$ or $\mathbf{F}_{p}, p>2$. Let $(i, j),\left(i^{\prime}, j^{\prime}\right)$ be such that $j \leq i, j^{\prime} \leq i^{\prime}$ and $2 i-j=2 i^{\prime}-j^{\prime} \geq 2$. Suppose $\nu \geq 2$. Then the composite

$$
H_{\mathrm{Zar}}^{j}\left(A, B / 2^{\nu}(i)\right)=H_{\text {ét }}^{j}\left(A, \mu_{2^{\nu}}^{\otimes i}\right) \stackrel{\beta^{i, j}}{\longrightarrow} K_{2 i-j}\left(A, \mathbf{Z} / 2^{\nu}\right) \stackrel{i^{\prime} c_{i^{\prime}, j^{\prime}}}{\longrightarrow} H_{\mathrm{Zar}}^{j^{\prime}}\left(A, B / 2^{\nu}\left(i^{\prime}\right)\right)
$$

is 0 if $\left(i^{\prime}, j^{\prime}\right) \neq(i, j)$ and is multiplication by $(-1)^{i-1} i$ ! if $(i, j)=\left(i^{\prime}, j^{\prime}\right)$. Here $\beta^{i, j}$ is the $(i, j)$-th component of the isomorphism $B_{A}^{2 i-j}$ of theorem 1.

Proof. By the proof of theorem $1 \mathrm{~b}$ ), the map $u_{A}^{2 i-j}$ of diagram (3.2) is surjective; hence the étale cohomology ring $H_{\text {ét }}^{*}\left(A, \mu_{2^{\nu}}^{\otimes *}\right)$ is generated by $H_{\text {ét }}^{0}\left(A, \mu_{2^{\nu}}\right)$ and $H_{\text {ét }}^{1}\left(A, \mu_{2^{\nu}}\right)$ up to transfer. By construction, the $\beta^{i, j}$ commute with product and transfer. By lemma 4.5, $i^{\prime} c_{i^{\prime}, j^{\prime}}$ commutes with transfer and, by [55, th. 3.2 (iv)], Soulé's product formula [42, th. 1]

$$
c_{i^{\prime}, j^{\prime}}(x \cdot y)=\sum_{\substack{i_{1}+i_{2}=i^{\prime} \\ i_{1} \geq m / 2, i_{2} \geq n / 2}}-\frac{\left(i^{\prime}-1\right) !}{\left(i_{1}-1\right) !\left(i_{2}-1\right) !} c_{i_{1}, j_{1}}(x) \cdot c_{i_{2}, j_{2}}(y)
$$

holds for any $(x, y) \in K_{m}\left(A, \mathbf{Z} / 2^{\nu}\right) \times K_{n}\left(A, \mathbf{Z} / 2^{\nu}\right)$ with $m+n=2 i-j$ since $A$ is local (in the formula, $j_{1}=2 i_{1}-m, j_{2}=2 i_{2}-n$ ). Here, if $m$ or $n$ is 1 , we use the Chern class of $(4.13)$, noting that $K_{1}(A) / 2^{\nu} \stackrel{\sim}{\rightarrow} K_{1}\left(A, \mathbf{Z} / 2^{\nu}\right)$. Thus we are reduced to the cases $(i, j)=(1,0)$ and $(i, j)=(1,1)$, which are trivial.

Corollary 5.3. Let $A, \nu$ be as in proposition 5.2. Then, for all $n \geq 2$, the kernel and cokernel of the map

$$
K_{n}\left(A, \mathbf{Z} / 2^{\nu}\right) \stackrel{\left(i c_{i, 2 i-n}\right)}{\longrightarrow} \prod_{i \geq 1} H_{\mathrm{Zar}}^{2 i-n}\left(A, B / 2^{\nu}(i)\right)
$$

are killed by $n$ !.

Proof. This follows from theorem 1 a) and proposition 5.2.

Corollary 5.4. For any $X$ and any $p \geq 0, n \geq 2$, the map

$$
H_{\mathrm{Zar}}^{p}\left(X, \prod_{i \geq 1} i c_{i, 2 i-n}\right)
$$

has kernel and cokernel killed by $n !$. 
Proof of theorem 3. We may assume that -1 is a square on $X$; the general case follows from this one by a transfer argument. Consider the morphism of spectral sequences from (5.1) to the direct product of all (5.2) $(k=2, i \geq 1)$ induced by all $i C_{i}^{(2)}, i \geq 1^{3}$. Note that, in this direct product, each $E_{r}^{p, q}$-term actually contains only a finite number of nonzero factors, and the same is true for the "abutment". Therefore, this direct product operation does not make convergence problems worse. By corollary 5.4, the induced map

$$
A_{2}^{p, q} \rightarrow \prod_{i \geq 1} B_{2}^{p, q}(i)
$$

has kernel and cokernel killed by $(2-q)$ !. The conclusion for $K_{n}^{T T}\left(X, \mathbf{Z} / 2^{\nu}\right), n \geq 3$ now follows from the following lemma, which is an easy consequence of computing two hypercohomology spectral sequences:

Lemma 5.5. Let $C \rightarrow D$ be a morphism of complexes. Assume that, for some $q$, the map $C^{i} \rightarrow D^{i}$ has kernel killed by $m_{i}$ and cokernel killed by $n_{i}$ for $i \in$ $\{q-1, q, q+1\}$, where $m_{i}, n_{i}$ are nonzero integers, except that $n_{q+1}$ is possibly 0 . Then the map $H^{q}\left(C^{\cdot}\right) \rightarrow H^{q}\left(D^{\cdot}\right)$ has kernel killed by $m_{q} n_{q-1}$ and cokernel killed by $m_{q+1} n_{q}$.

Note that this lemma takes care of the fringe effect as, by definition, $A_{r+1}^{p,-p}$ is a subgroup of $H^{p,-p}\left(A_{r}\right)$, and similarly for the $B_{r}(n)$.

If $X$ is smooth over a field or a dvr, then $H_{\text {Zar }}^{p}\left(X, \mathcal{H}^{q}\left(B / 2^{\nu}(n)\right)\right)=0$ for $p>q$ by Gersten's conjecture, hence the fringe effect disappears (compare [7, th. 3 and remark 1 p. 290]) and we can extend the conclusion to $n=2$.

\section{REFERENCES}

[1] H. Bass, M. P. Murthy Grothendieck groups and Picard groups of abelian group rings, Ann. Math. 86 (1967), 16-73.

[2] A. A. Beilinson Height pairings between algebraic cycles, Lect. Notes in Math. 1289, Springer, 1987, 1-26.

[3] S. Bloch Algebraic cycles and higher K-theory, Adv. Math. 61 (1986), 267-304.

[4] S. Bloch, S. Lichtenbaum A spectral sequence for motivic cohomology, preprint, 1995.

[5] S. Bloch, A. Ogus Gersten's conjecture and the homology of schemes, Ann. Sci. Ec. Norm. Sup., 4. sér. 7 (1974), 181-202.

[6] W. Browder Algebraic K-theory with coefficients $\mathbf{Z} / p$, Lect. Notes in Math. 657, Springer, 1978, 80-130.

[7] K. Brown, S. Gersten Algebraic K-theory as generalized sheaf cohomology, Lect. Notes 341, Springer, 1973, 266-292.

[8] J.-L. Colliot-Thélène, R. T. Hoobler, B. Kahn The Bloch-Ogus-Gabber theorem, Fields Inst. Comm. 16, AMS, 1997, 31-94.

[9] J.-L. Colliot-Thélène, J.-J. Sansuc, C. Soulé Torsion dans le groupe de Chow de codimension 2, Duke Math. J. 50 (1983), 763-801.

\footnotetext{
${ }^{3}$ As all simplicial sheaves are sheaves of $H$-spaces, it makes sense to multiply $C_{i}^{(2)}$ by an integer.
} 
[10] P. Deligne, Séminaire de Géométrie algébrique du Bois-Marie (SGA 4 1/2), Lect. Notes in Math. 569, Springer Verlag, 1977.

[11] W. Dwyer, E. Friedlander Algebraic and étale K-theory, Trans. A.M.S. 292 (1985), 247-280.

[12] W. Dwyer, E. Friedlander, V. Snaith, R. Thomason Algebraic K-theory eventually surjects onto topological K-theory, Invent. Math. 66 (1982), 481-491.

[13] P. Elbaz-Vincent, S. Müller-Stach Milnor K-theory of rings, higher Chow groups and applications, preprint, 1998.

[14] H. Esnault, B. Kahn, M. Levine, E. Viehweg The Arason invariant and mod 2 algebraic cycles, J. A.M.S. 11 (1998), 73-118.

[15] B. Feigin, B. Tsygan Additive K-theory, Lect. Notes in Math. 1289, Springer, New York, 1987, 67-209.

[16] O. Gabber Affine analog of the proper base change theorem, Israel J. Math. 87 (1994), 325335.

[17] O. Gabber K-theory of henselian local rings and henselian pairs, Contemp. Math. 126, 5970, AMS, 1992.

[18] O. Gabber, letter to the author, February 18, 1998.

[19] S. Geller, C. Weibel Hodge decompositions of Loday symbols in $K$-theory and cyclic homology, $K$-theory 8 (1994), 587-632.

[20] T. Geisser. M. Levine The Bloch-Kato conjecture and a theorem of Suslin-Voevodsky, preprint, 1998.

[21] H. Gillet Riemann-Roch theorems for higher algebraic K-theory, Adv. Math. 40 (1981), 203289.

[22] H. Gillet, in preparation.

[23] H. Gillet, M. Levine The relative form of Gersten's conjecture over a discrete valuation ring: the smooth case, J. Pure Appl. Algebra 46 (1987), 59-71.

[24] R. Hoobler The Merkuriev-Suslin theorem for arbitrary semi-local rings, preprint, 1996.

[25] R. de Jeu On $K_{4}^{(3)}$ of curves over number fields, Invent. Math. 125 (1996), 523-556.

[26] B. Kahn Some conjectures in the algebraic K-theory of fields, I: K-theory with coefficients and étale K-theory, NATO ASI Series, Ser. C. 279, Kluwer, 1989, 117-176.

[27] B. Kahn Deux théorèmes de comparaison en cohomologie étale; applications, Duke Math. J. 69 (1993), 137-165.

[28] B. Kahn On the Lichtenbaum-Quillen conjecture, NATO ASI Series, Ser. C. 407, Kluwer, 1993, 147-166.

[29] B. Kahn Bott elements in algebraic K-theory, Topology 36 (1997), 963-1006.

[30] B. Kahn l-adic cohomology, motivic cohomology and the Tate conjecture, preprint, 1999.

[31] K. Kato A Hasse principle for two-dimensional global fields, J. reine angew. Math. 366 (1986), 142-183.

[32] M. Levine Techniques of localization in the theory of algebraic cycles, preprint, 1999.

[33] M. Levine K-theory and motivic cohomology of schemes, preprint, 1999.

[34] F. Morel, V. Voevodsky $\mathbf{A}^{1}$-homotopy category of schemes, preprint, 1998.

[35] Y. Nisnevich, The completely decomposed topology on schemes and the associated descent spectral sequences in algebraic $K$-theory, in Algebraic $K$-theory: connections with geometry and topology, J.F. Jardine, V.P. Snaith, eds., NATO ASI Series, Sec. C 279 (1989), 241-342.

[36] D. Quillen Higher algebraic K-theory, I, Lecture Notes in Mathematics 341, Springer, Berlin, 1973, 77-139.

[37] D. Quillen Higher algebraic K-theory, Proc. Intern. Congress Math. (I), Vancouver, 1974, 171-176. 
[38] L. Reid $N$-dimensional rings with an isolated singular point having nonzero $K_{-N}, K$-theory 1 (1987), 197-206.

[39] J. Rognes, C. Weibel, Two-primary algebraic K-theory of rings of integers in number fields, preprint, 1997.

[40] M. Rost Chow groups with coefficients, Doc. Math. 1 (1996), 319-393.

[41] J.-P. Serre Cohomologie galoisienne (new edition), Lect. Notes in Math. 5, Springer, 1994.

[42] C. Soulé $K$-théorie des anneaux d'entiers de corps de nombres et cohomologie étale, Invent. Math. 55 (1979), 251-295.

[43] R. Strano On the étale cohomology of Hensel rings, Comm. in Alg. 12 (1984), 2195-2211.

[44] A. Suslin On the K-theory of local fields, J. Pure Appl. Algebra 34 (1984), 301-318.

[45] A. Suslin Algebraic K-theory of fields, Proceedings of the International Congress of Mathematicians, Berkeley, 1986, 222-244.

[46] A. Suslin Higher Chow groups and étale cohomology, preprint, 1994.

[47] A. Suslin, V. Voevodsky Bloch-Kato conjecture and motivic cohomology with finite coefficients, preprint, 1995.

[48] R. Thomason Algebraic K-theory and étale cohomology, Ann. Sci. Ec. Norm. Sup. 4e sér. 13 (1985), 437-452.

[49] R. Thomason Bott stability in algebraic K-theory, Contemp. Math. 55 (I), AMS, 1986, 389406.

[50] R. Thomason Survey of algebraic vs. topological K-theory, Contemp. Math. 83, AMS, 1989, 393-443.

[51] R. Thomason, T. Trobaugh Higher algebraic K-theory of schemes and of derived categories, The Grothendicek Festschrift II, Progress in Math., Birkhäuser, 1990, 247-435.

[52] V. Voevodsky The Milnor conjecture, preprint, 1996.

[53] C. Weibel K-theory and analytic isomorphisms, Invent. math. 61 (1980), 177-197.

[54] C. Weibel Pic is a contracted functor, Invent. Math. 103 (1991), 351-377.

[55] C. Weibel Etale Chern classes at the prime 2, NATO ASI Series, Ser. C 407, Kluwer, 1993, 249-286.

[56] C. Weibel The $K$-theory of normal surfaces, in preparation.

[EGA4] A. Grothendieck, J. Dieudonné, Éléments de géométrie algébrique, chapitre IV: Étude locale des schémas et des morphismes de schémas (2ème partie), Publ. Math. IHES 24, 1965.

[SGA4] A. Grothendieck, M. Artin, J.-L. Verdier Théorie des topos et cohomologie étale des schémas (SGA4), Vol. 3, Lect Notes in Math. 305, Springer, 1971.

Institut de Mathématiques de Jussieu, Equipe Théories Géométriques, Université Paris 7, Case 7012, 75251 Paris Cedex 05, France

E-mail address: kahn@math.jussieu.fr 